\title{
Anisotropic exciton excitations and optical properties of Hittorf's phosphorene
}

\author{
Ju Zhou $\odot,{ }^{1}$ Tian-Yi Cai, ${ }^{1, *}$ and Sheng Ju ${ }^{1,2,3, \dagger}$ \\ ${ }^{1}$ Department of Physics and Jiangsu Key Laboratory of Thin Films, Soochow University, Suzhou 215006, People's Republic of China \\ ${ }^{2}$ Department of Physics, University of California, Berkeley, Berkeley, California 94720, USA \\ ${ }^{3}$ Materials Sciences Division, Lawrence Berkeley National Laboratory, Berkeley, California 94720, USA
}

(Received 10 April 2020; accepted 28 July 2020; published 21 August 2020)

\begin{abstract}
Due to the exciton effects, two-dimensional materials have displayed strong light-matter interactions. Here, by considering many-body effects of electron-electron and electron-hole interactions, we provide a more accurate quasiparticle electronic structure, exciton, and optical absorption spectrum of Hittorf's phosphorene, a monolayer of Hittorf's (or violet) phosphorus obtained recently via exfoliation method. The fibrous nature of the crystal structure with twofold rotation symmetry leads to anisotropic electron-hole excitations, and an obvious dichroism in optical absorption is revealed over a wide spectral range. The first exciton state located at $2.4 \mathrm{eV}$ is bright with a large binding energy of $0.9 \mathrm{eV}$ and a lifetime of $18 \mathrm{~ns}$. These unusual excitonic and optical properties demonstrate its potential applications in optoelectronics.
\end{abstract}

DOI: 10.1103/PhysRevResearch.2.033288

\section{INTRODUCTION}

Since the discovery of graphene, two-dimensional (2D) materials have attracted intense interest in photonics and optoelectronics [1-3]. Compared with traditional electronic and photonic materials, 2D materials exhibit many advantages. Their surfaces are naturally passivated without any dangling bonds, making it easier to integrate them with current electronic and photonic devices without conventional lattice mismatch problems. In addition, the quantum confinement in the direction perpendicular to the 2D plane leads to enhanced electron-electron interaction and consequently distinct electronic properties from their bulk parent materials. The strong electron-hole interaction, on the other hand, leads to strong light-matter interaction, and, despite being atomically thin, most 2D materials absorb photons strongly [4-23]. As shown in Fig. 1, from gapless graphene to narrow-band-gap black phosphorene, intermediate-band-gap transition-metal dichalcogenides, and wide-band-gap semiconductors of blue phosphorene, III-VI monochalcogenides, and boron nitride, 2D materials could cover a wide range of the optical spectrum, which makes them useful in the applications of photodetectors, photovoltaics [24], light-emitting-diodes (LEDs), etc.

Unlike other 2D materials, e.g., transition-metal dichalcogenides and III-VI monochalcogenides, the unique crystal structure of black phosphorus gives rise to an obvious anisotropic electron-hole excitation, with 1.6-eV difference in the optical absorption edge between the polarizations along the zigzag and armchair directions of 2D puckered structure

\footnotetext{
*caitianyi@suda.edu.cn

†jusheng@suda.edu.cn
}

Published by the American Physical Society under the terms of the Creative Commons Attribution 4.0 International license. Further distribution of this work must maintain attribution to the author(s) and the published article's title, journal citation, and DOI.
[13-19]. The anisotropy is intrinsic and independent of layer thickness and environment screening, showing robust application in optoelectronic devices. In nature, phosphorus exhibits many allotropes [25]. At high pressures, black phosphorus will undergo a transition to A7 phase [26-28], the monolayer of which was recently named as blue phosphorene $[29,30]$ and synthesized successfully with the help of bottom-up growth techniques on metallic substrates [31-36]. Unlike black phosphorene, this 2D buckled hexagonal crystal structure exhibits a much larger band gap of $3.41 \mathrm{eV}$ and the first exciton state at $2.85 \mathrm{eV}$ (see Appendix A). However, the indirect band gap prohibits its wide applications in optoelectronics. On the other hand, violet (or Hittorf's) phosphorus is another allotrope revealed long ago [37]. The fibrous and layered structure therein distinguishes it from other phosphorus allotropes [38,39]. $A b$ initio calculations showed that its monolayer is a wide-bandgap semiconductor with a direct band gap of approximately $2.5 \mathrm{eV}$ [40]. High carrier mobility was also predicted to be comparable with black phosphorene [41-44]. More importantly, total-energy calculations indicated that the exfoliation of a monolayer from bulk is similar to black phosphorus [40]. Recently, under ambient conditions, the monolayer of violet (or Hittorf's) phosphorus was realized experimentally with both mechanical and solution exfoliation methods [45]. In particular, the high quality of the solution exfoliated sample paves a solid foundation for further exploration of its physical and chemical properties [45]. From the crystal structure of Hittorf's phosphorus monolayer (see Fig. 2), the 2D system consists of phosphorus fibers making up two sets of sublayers, each bonded covalently with neighboring fibers of another sublayer. The monolayer contains fibers cross-hatched in two sublayers with a total of 42 atoms in the unit cell. The bulk Hittorf's phosphorus displays a monoclinic structure, with the lattice constants of $a=9.21 \AA, b=9.15 \AA, c=22.60 \AA$, and $\beta=106.1^{\circ}$ [38]. Although Hittorf's phosphorene is believed to be a wide-band-gap semiconductor [40], the accurate quasiparticle band gap, exciton, and optical properties 


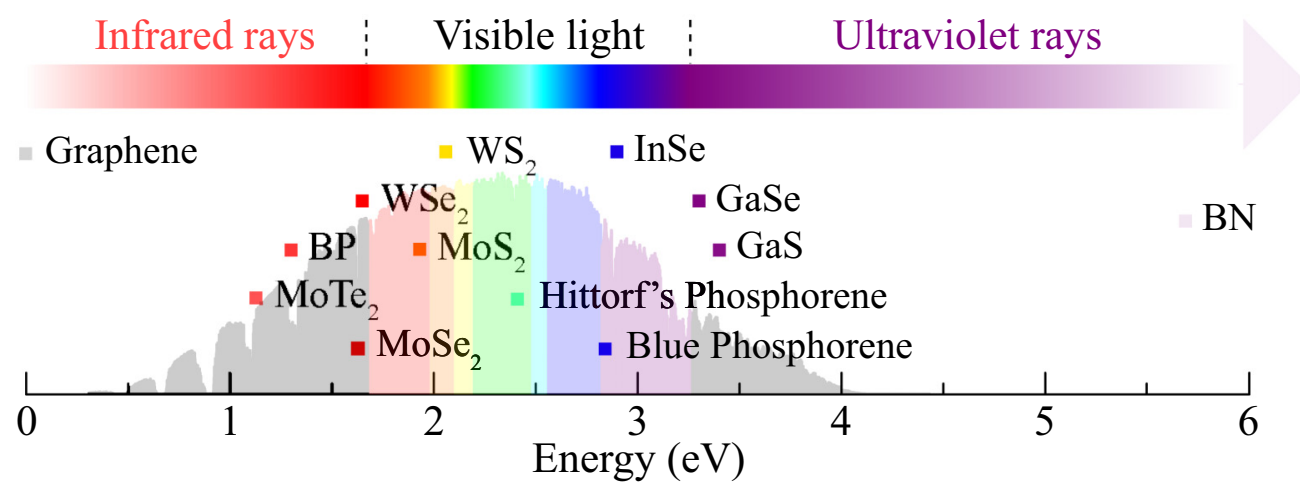

FIG. 1. 2D materials covering a broad spectral range, including zero-band-gap graphene, narrow-band-gap black phosphorene, intermediate-band-gap transition-metal dichalcogenides, and wide-band-gap Hittorf's phosphorene, blue phosphorene, III-VI monochalcogenides, and hexagonal boron nitride. The solar spectrum is also shown [24].

are still lacking. Many-body effects arising from electronelectron and electron-hole interactions are very important in low-dimensional systems. In particular, the unique dielectric screening and quantum confinement effect are crucial for the excited-state properties in 2D materials [46-50]. In this paper, based on accurate ab initio $G W$-BSE (Bethe-Salpeter equation) method, we have studied the quasiparticle electronic structure, exciton, and optical properties of 2D Hittorf's phosphorene. A direct band gap of $3.32 \mathrm{eV}$ has been identified. The optical spectrum is dominated by exciton effects. The first exciton state is located at $2.41 \mathrm{eV}$ with a strong binding energy of $0.91 \mathrm{eV}$. Furthermore, strong polarization direction dependence of electron-hole excitations has been revealed in this 2D material. These results have shown the potential applications of Hittorf's phosphorene in optoelectronics.

\section{COMPUTATIONAL METHOD}

Our first-principles calculations are performed using density-functional theory (DFT) with the generalized gradient approximation as implemented in the QUANTUM ESPRESSO package [51,52]. The quasiparticle band structures are

calculated with the BERKELEYGW package [53-55]. A slab model is used with a vacuum layer of $20 \AA$ along the out-ofplane direction. Structure optimization starts from the atomic positions and in-plane lattice constants extracted from bulk Hittorf's phosphorus [38]. The detailed crystal structures are given in Appendix B. In the meantime, a truncated Coulomb interaction between Hittorf's phosphorene and its periodic image is adopted. The quasiparticle self-energies are obtained by solving the following Dyson equation:

$$
\left[-\frac{1}{2} \nabla^{2}+V_{\text {ion }}+V_{\mathrm{H}}+\Sigma\left(E_{n \mathbf{k}}^{\mathrm{QP}}\right)\right] \psi_{n \mathbf{k}}^{\mathrm{QP}}=E_{n \mathbf{k}}^{\mathrm{QP}} \psi_{n \mathbf{k}}^{\mathrm{QP}},
$$

where $\Sigma$ is the self-energy operator within the $G W$ approximation, and $E_{n \mathbf{k}}^{\mathrm{QP}}$ and $\psi_{n \mathbf{k}}^{\mathrm{QP}}$ are the quasiparticle energies and wave functions, respectively. We use the $G_{0} W_{0}$ level with the generalized plasma pole model. The electron-hole excitations are then calculated by solving the BSE for each exciton state $S$ :

$$
\left(E_{c \mathbf{k}}^{\mathrm{QP}}-E_{v \mathbf{k}}^{\mathrm{QP}}\right) A_{v c \mathbf{k}}^{S}+\sum_{v^{\prime} c^{\prime} \mathbf{k}^{\prime}}\left\langle v c \mathbf{k}\left|K^{\mathrm{eh}}\right| v^{\prime} c^{\prime} \mathbf{k}^{\prime}\right\rangle=\Omega^{S} A_{v c \mathbf{k}}^{S},
$$
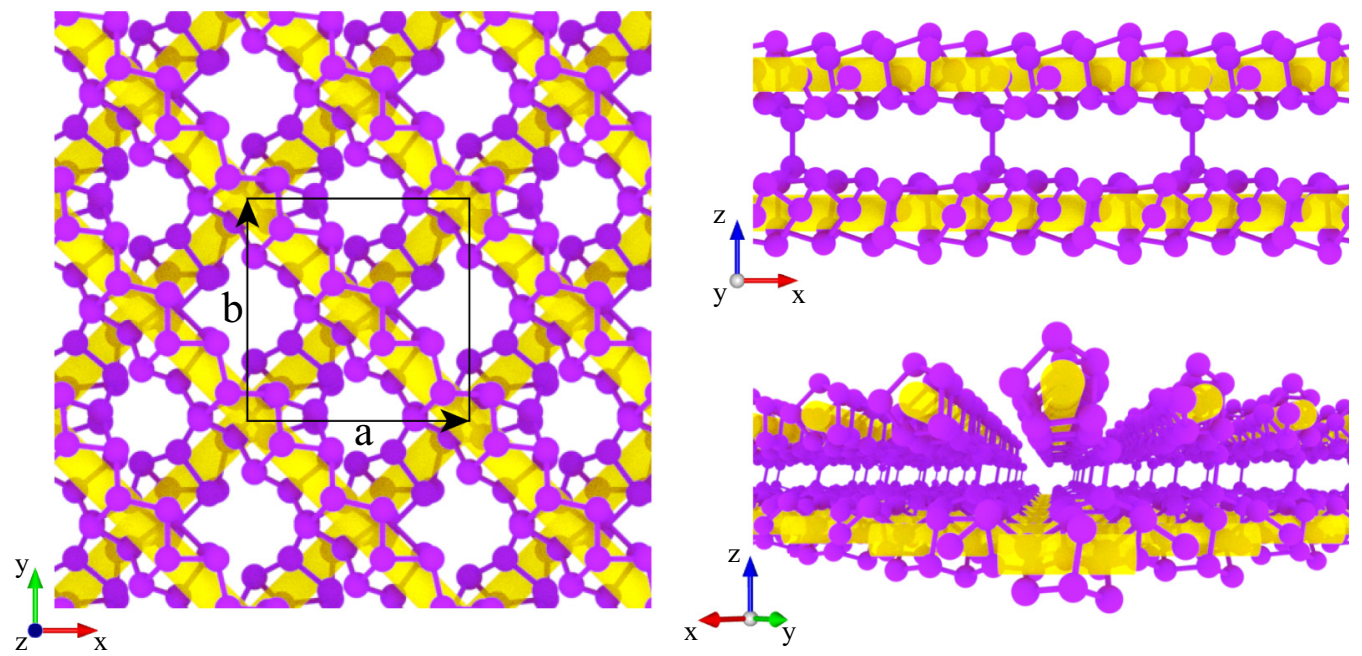

FIG. 2. Crystal structure of 2D Hittorf's phosphorene. Violet spheres represent phosphorus atoms, while the yellow tubes are added as guides to the eye only to emphasize the fibrous nature of Hittorf's phosphorene. The unit cell is shown as black solid lines in the top view. 


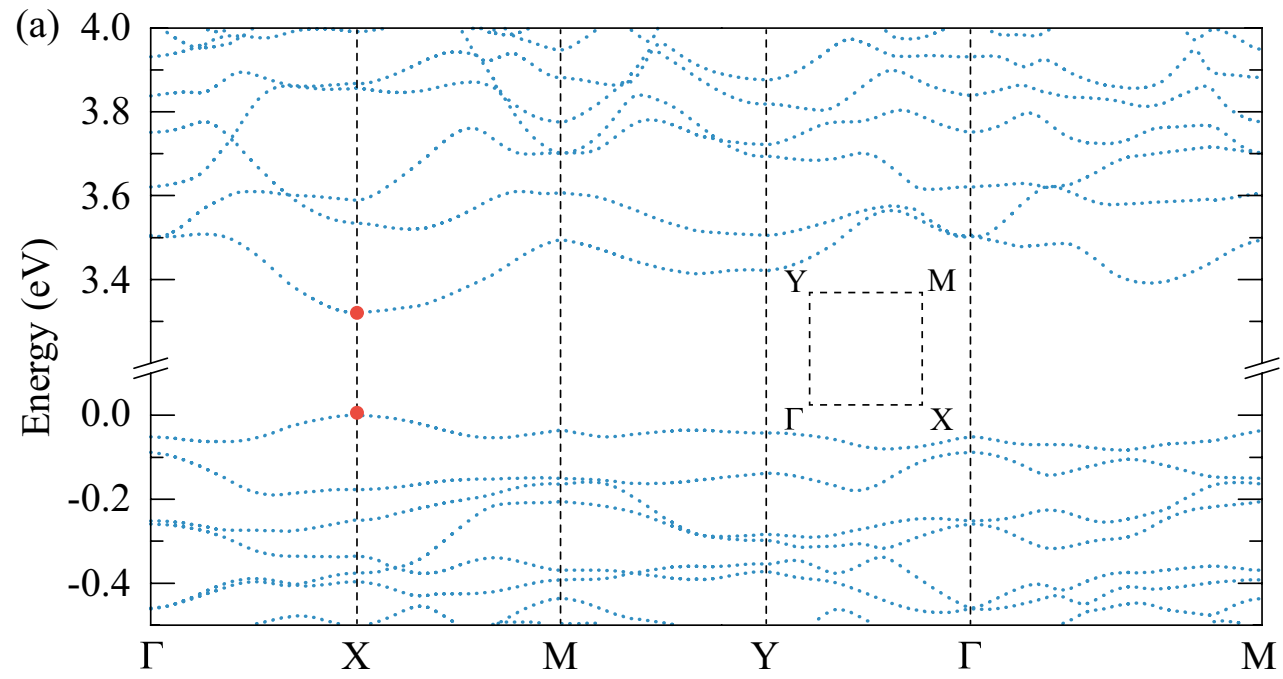

(b)

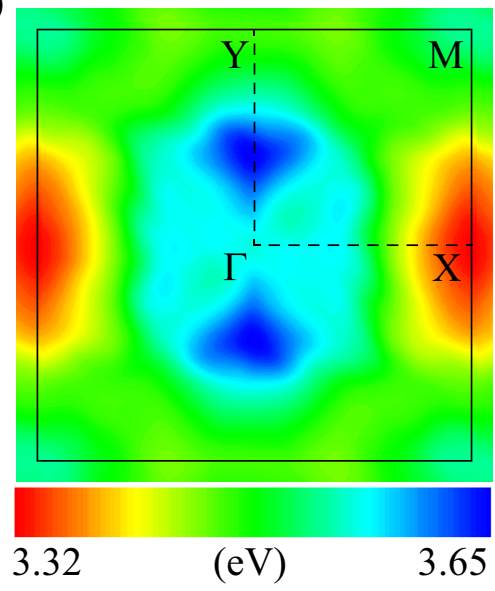

(c)

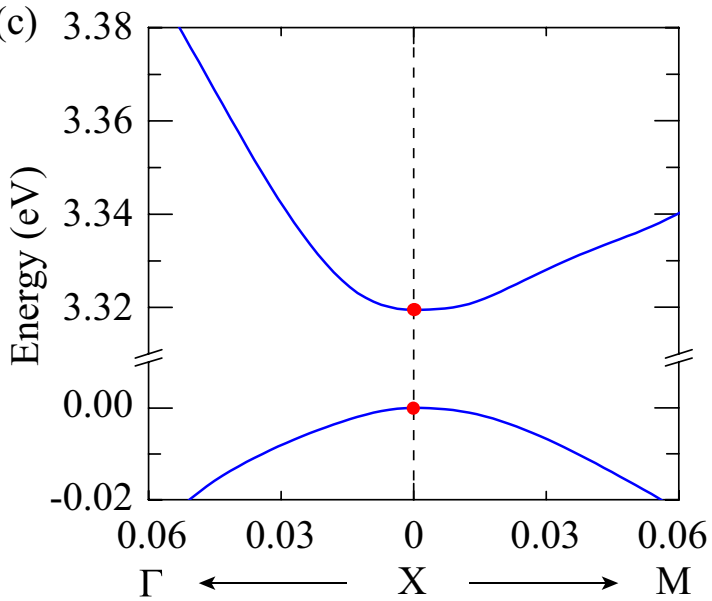

FIG. 3. (a) Quasiparticle electronic band structure of 2D Hittorf's phosphorene. (b) Direct band gap in Hittorf's phosphorene for the whole BZ. (c) Quasiparticle band structure around the band edge at the $X$ point.

where $A_{v c \mathbf{k}}^{S}$ is the exciton wave function, $\Omega^{S}$ is the excitation energy, and $K^{\mathrm{eh}}$ is the electron-hole interaction kernel. Finally, we obtain the imaginary parts of frequency dependent complex dielectric function $\epsilon_{2}(\omega)$ as

$$
\epsilon_{2}(\omega)=\frac{16 \pi^{2} e^{2}}{\omega^{2}} \sum_{S}|\mathbf{e} \cdot\langle 0|\mathbf{v}| S\rangle|^{2} \delta\left(\omega-\Omega^{S}\right),
$$

where $\mathbf{v}$ is the velocity operator and $\mathbf{e}$ is the polarization of the incoming light. Here, we use absorbance $A$ of $2 \mathrm{D}$ materials to measure its optical properties, which is expressed as

$$
A(\omega)=1-e^{-\alpha(\omega) d}=1-e^{-\frac{\omega \epsilon_{2} d}{\hbar c}},
$$

where $\alpha$ is the absorption coefficient, $d$ is the thickness of the simulation cell along the direction perpendicular to the layer, $\epsilon_{2}$ is the imaginary part of the dielectric function, and $\omega$ is the photon energy. We employ norm-conserving pseudopotentials with a plane-wave cutoff of $60 \mathrm{Ry}$ [56]. For the convergence of quasiparticle energies [57], we have tested the dependence on $k$-grid size, number of bands, as well as dielectric cutoff (see Appendix C). We use a coarse $k$ grid of $6 \times 6 \times 1$, empty bands of 30 times more than the valence bands, and the dielectric cutoff of $10 \mathrm{Ry}$. For the BSE part, a fine $k$ grid of $36 \times 36 \times 1$ is used (see Appendix C). It is noted that fine sampling is necessary to capture fast variation in screening at small wave vectors and fine features in exciton wave functions, which are tightly localized in $k$ space [5]. We use a Gaussian smearing with a broadening constant of $5 \mathrm{meV}$ in the optical absorbance spectrum. The number of bands for optical transitions is 8 for both valence and conduction bands, which is sufficient to cover the span of the visible light.

\section{RESULTS AND DISCUSSION}

As indicated by the quasiparticle band structure in Fig. 3(a), this monolayer of Hittorf's phosphorene is a directband-gap semiconductor. The direct quasiparticle band gap of this 2D material is shown in Fig. 3(b) and the band structure near the band edge is displayed in Fig. 3(c). The band gap at the $X$ point is $3.32 \mathrm{eV}$. In Table I, we list the band gap obtained with other approaches [40,45]. At other $k$ points in the Brillouin zone (BZ), the band gap increases a little to the highest value of $3.65 \mathrm{eV}$, indicating a small variation of band gap and a possible strong optical absorption from uniform electron-hole excitations. Also, the zoomed-in band structures of the bottom of the conduction band and 
TABLE I. Band gap of Hittorf's phosphorene from different approaches.

\begin{tabular}{lc}
\hline \hline & $E_{g}(\mathrm{eV})$ \\
\hline Present work & $1.93(\mathrm{PBE}), 3.32\left(G_{0} W_{0}\right), 2.41(\mathrm{BSE})$ \\
Ref. [40] & $1.93(\mathrm{PBE}), 2.71(\mathrm{HSE})$ \\
Ref. [45] & $2.54(\mathrm{HSE})$ \\
\hline \hline
\end{tabular}

the top of the valence band indicate that both electrons and holes near the band edge are much heavier along the $X-M$ direction than along the $\Gamma-X$ direction. Such an anisotropy arises from the inherent bilayer structure, where as indicated in Fig. 2 twofold rotation symmetry associated with point group $C_{2}$ is only found along the $x$ axis. At the $X$ point, the effective mass (relative to $m_{0}$ and fitting within 0.05 bohr $^{-1}$ ) of electrons is 0.7 along the $\Gamma-X$ direction and the value reaches as high as 3.8 along the $X-M$ direction. For the hole, they are 1.1 and 2.4 , respectively. Due to the one-dimensional (1D) fibrous nature of the crystal structure of Hittorf's phosphorus, the overall band structure shows relatively less dispersion. In the meantime, electrons spread over two sublayers, while holes are mostly concentrated at interlayer space (see Fig. 4).

As shown in Fig. 5, similar to other 2D materials, the excitonic effect is obvious in the optical spectrum of 2D Hittorf's phosphorene. For such a wide-band-gap semiconductor, the optical absorption spectrum obtained by considering electron-hole interactions is totally different from that based on independent particle approximation. The first absorption peak is located at $2.41 \mathrm{eV}$, which is of green light. On the other hand, the main absorption is located around $3.0 \mathrm{eV}$, coinciding with the solar spectrum, and therefore showing its potential application in photovoltaics [58]. The binding energy of the first exciton state is around $0.9 \mathrm{eV}$, which is much larger than the value of $0.6 \mathrm{eV}$ revealed in the $\mathrm{MoS}_{2}$ single layer [11], and $0.5 \mathrm{eV}$ in black phosphorene [18]. It is noted that the band gap is also larger here (3.3 eV compared with $2.6 \mathrm{eV}$ for the $\mathrm{MoS}_{2}$ single layer and $2.1 \mathrm{eV}$ in black phosphorene). The strong binding energy in 2D materials is due to its reduced dimension, where the Coulomb interactions could not be effectively screened. Compared with the $\mathrm{MoS}_{2}$ monolayer with a thickness of $3.13 \AA$ and black phosphorene with a thickness of $1.24 \AA$, the effective thickness of Hittorf's phosphorene is much larger, and reaches as high as $10.06 \AA$. However, the 1D fibrous nature of the crystal structure provides an effective antiscreening of Coulomb interaction [59], and consequently possible large binding energy. Our first-principles prediction of a strong bound exciton is also in good agreement with model calculations. The detailed calculations can be found in Appendix D. In Figs. 6 and 7, we show the detailed quasiparticle band structure and optical properties of the 1D phosphorus nanotube, respectively. The band edge is located at the $X$ point and the direct band gap therein is $4.77 \mathrm{eV}$. The orbitals spread almost uniformly along the tube with $3 p$ characteristics [see Fig. 6(b)]. From the optical absorption shown in Fig. 7(a), the spectrum is redshifted a lot compared with the independent particle approximation and the binding energy of the first exciton state reaches as high as $2.15 \mathrm{eV}$. The detailed exciton states are shown in Fig. 7(b), indicating the dark nature of the first exciton.

The detailed energies of exciton states in 2D Hittorf's phosphorene are shown in Fig. 8(b), where the bright excitons are indicated by red lines and the dark excitons are indicated by gray lines. Here, the dark excitons have oscillation strength less than $10^{-5}$. For example, the first dark exciton at $2.63 \mathrm{eV}$ has an oscillation strength of $10^{-8}$, which corresponds to the optical absorbance of less than $0.01 \%$. Similar to other
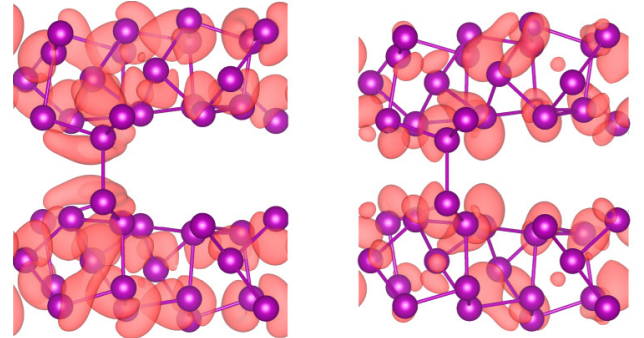

$\mathrm{CB}-\Gamma$

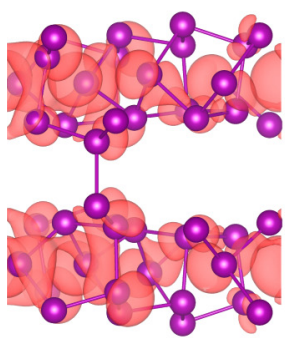

$\mathrm{VB}-\Gamma$

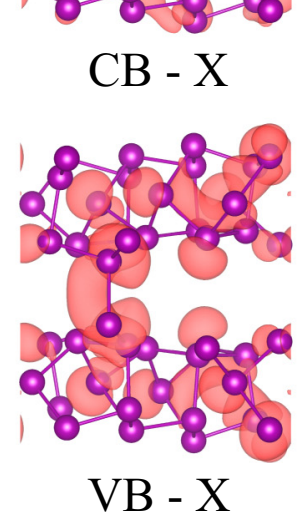

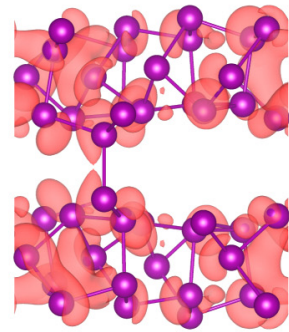

$\mathrm{CB}-\mathrm{M}$

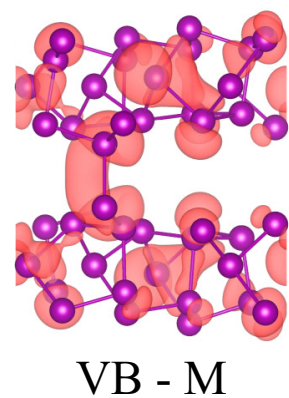

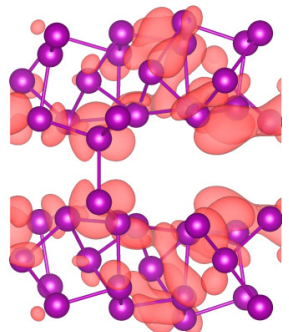

$\mathrm{CB}-\mathrm{Y}$

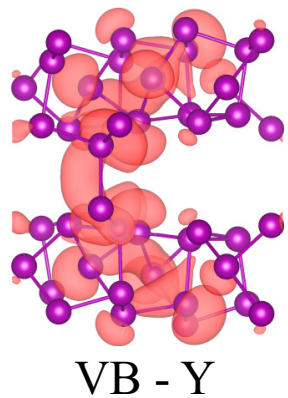

FIG. 4. Electronic charges at different $k$ points of the top of the valence band (VB) and the bottom of the conduction band (CB) in Hittorf's phosphorene. 


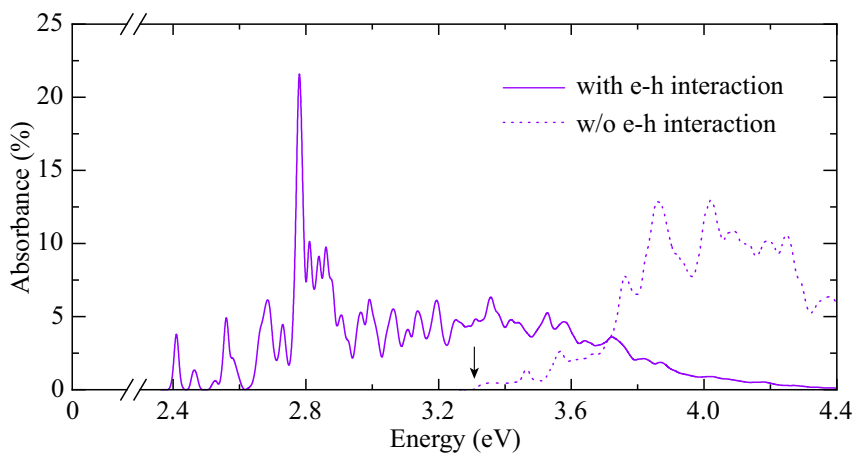

FIG. 5. Optical absorbance (averaged over the electric polarizations along $x$ and $y$ directions) in 2D Hittorf's phosphorene with and without the inclusion of electron-hole interactions. The arrow indicates the quasiparticle band gap.

2D materials, these discrete exciton levels do not obey the hydrogen model for the unique $2 \mathrm{D}$ dielectric screening and 2D band structure [5].

Compared with 2D black phosphorene with a giant optical anisotropy of $1.6 \mathrm{eV}$, the difference in the absorption edges for two orthogonal directions is just around $0.06 \mathrm{eV}$. However, as shown in Fig. 8(a), when switching the direction of polarization of light from the $x$ axis $\left(0^{\circ}\right)$ to the $y$ axis $\left(90^{\circ}\right)$, the spectrum associated with each excitonic state shows strong oscillations in absorption strength. Clearly, the electron-hole pairs are excited anisotropically. It is also noted that the optical absorption edges are different between two orthogonal

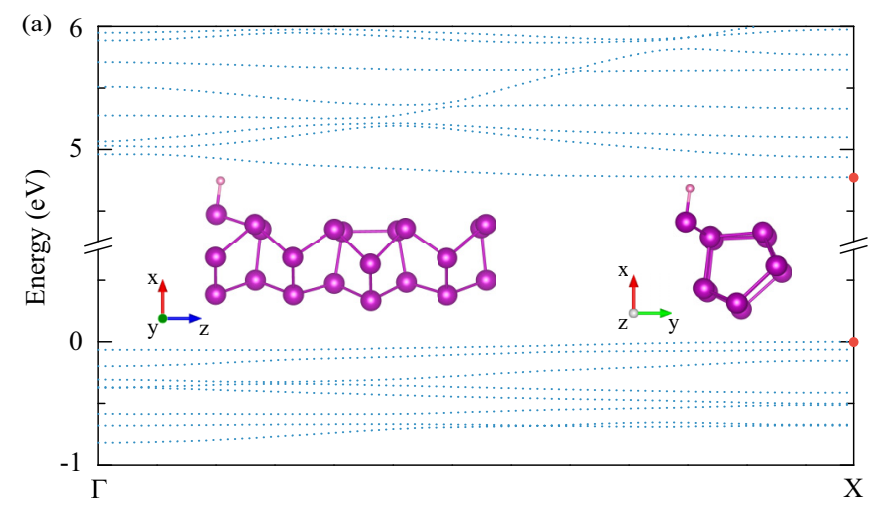

(b)
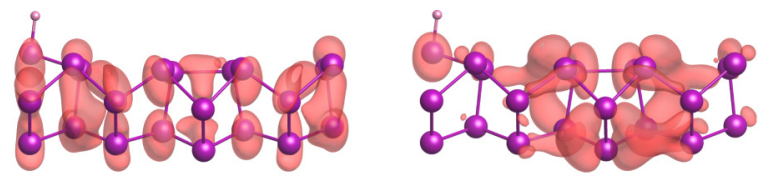

$\mathrm{CB}-\Gamma$

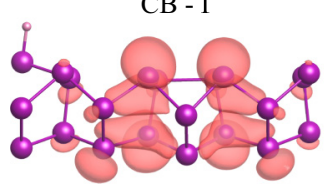

VB $-\Gamma$

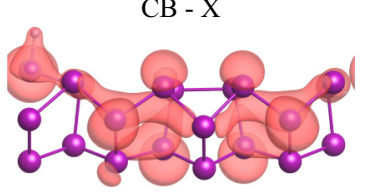

VB - X

FIG. 6. (a) Quasiparticle electronic band structure of the 1D phosphorus nanotube. The inset shows its crystal structure. (b) Electronic charges at different $k$ points of the top of the valence band (VB) and the bottom of the conduction band (CB).
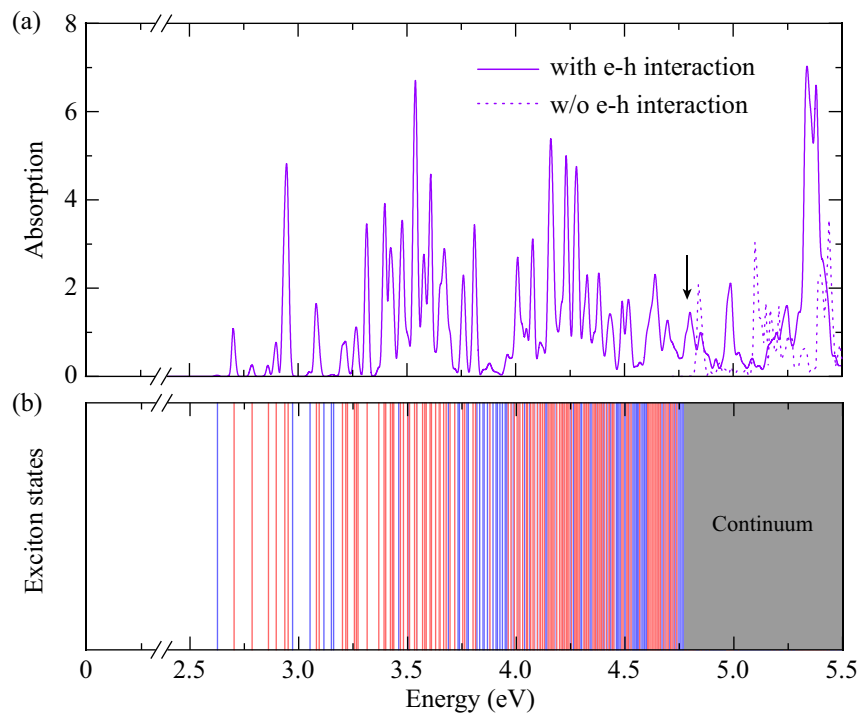

FIG. 7. (a) Optical absorption in the 1D phosphorus nanotube with and without the inclusion of electron-hole interactions. The arrow indicates the quasiparticle band gap. (b) Exciton spectrum. Red lines are for the bright exciton states and blue lines are for the dark exciton states.

directions of polarization when the electron-hole interaction is not included (see Appendix E). The excitonic effect amplifies the optical anisotropy further in this $2 \mathrm{D}$ system.

Besides the first exciton state, we further consider the following bright exciton states, which are located at 2.465 , 2.526, 2.555, 2.562, and $2.579 \mathrm{eV}$, respectively. In Fig. 9, we show a real-space plot of these exciton states. It is found that the electrons are distributed along tubes, when we fix the hole at the center of two sublayers that has the maximum density for the hole states (as indicated in Fig. 4).
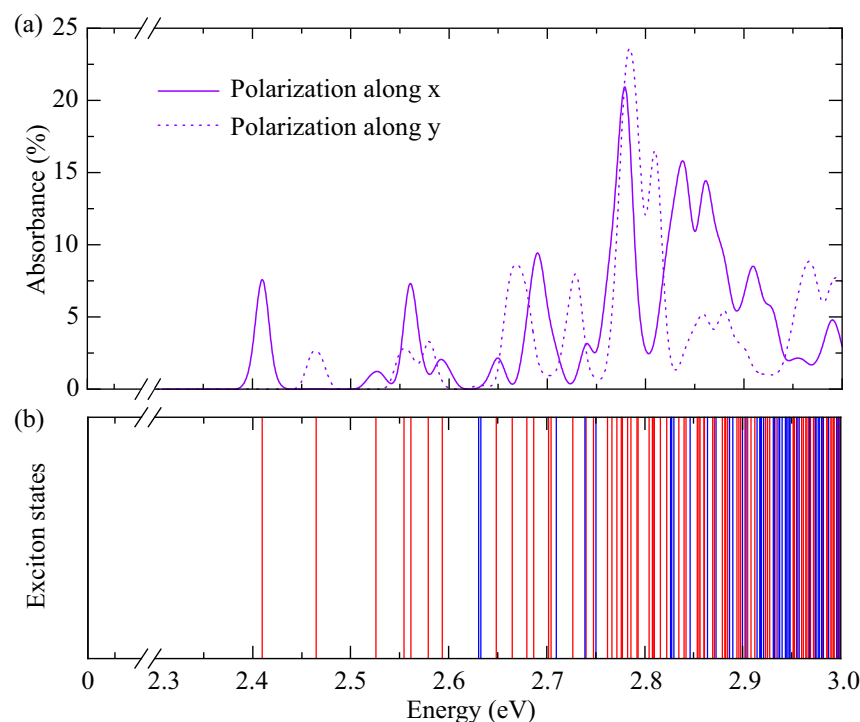

FIG. 8. (a) Optical absorbance in 2D Hittorf's phosphorene for different polarization directions. (b) Exciton spectrum. Red lines are for the bright exciton states and blue lines are for the dark exciton states. 

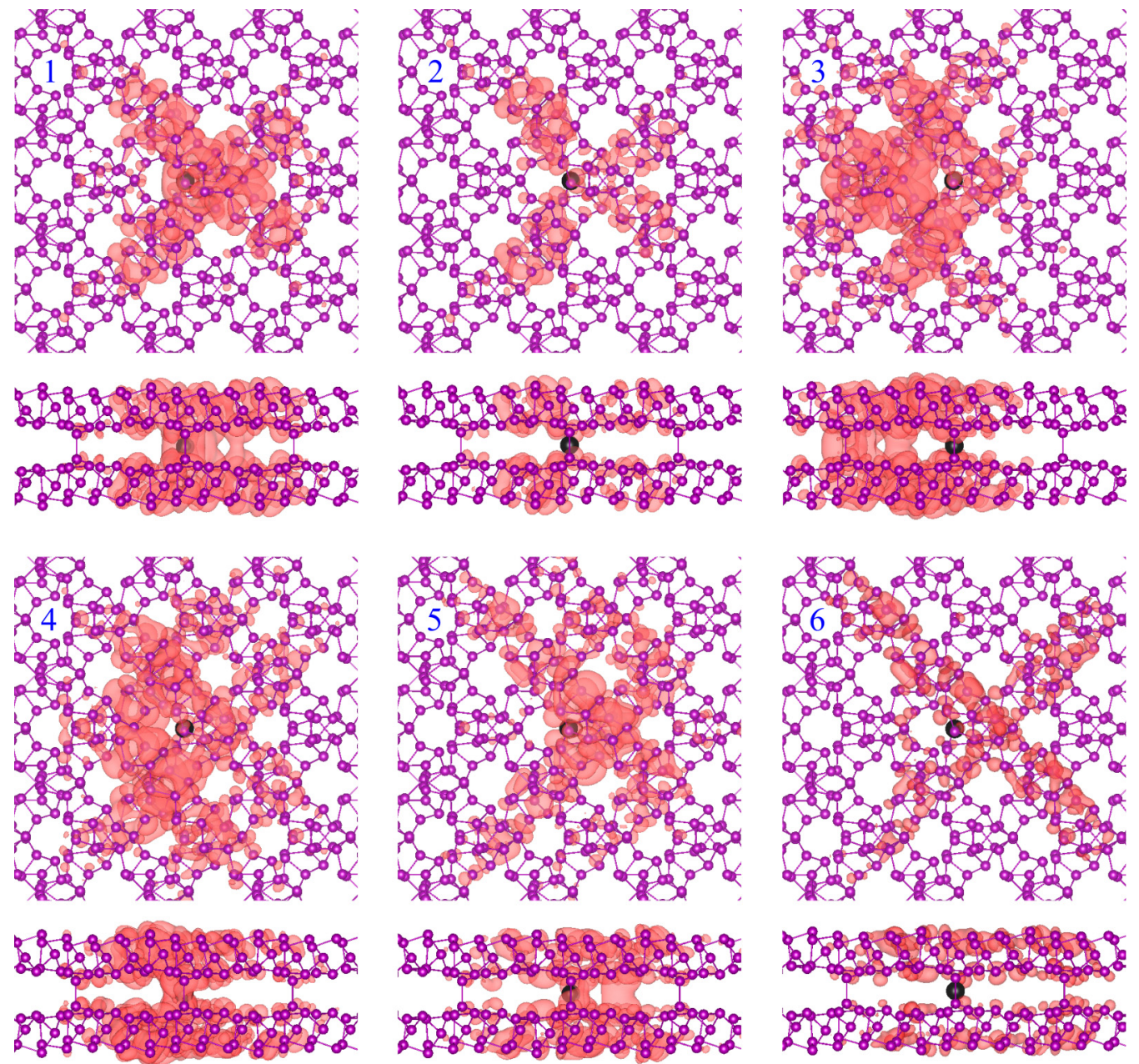

FIG. 9. Real-space modulus squared of the wave function of the first six excitons in 2D Hittorf's phosphorene. The black dot indicates the hole position that has the maximum density for the hole states.

Electron-hole excitations are highly localized on the two orthogonally crossed tubes for the first and the second excitons. For the next excited states, the electrons are either delocalized further to the neighboring tubes of both upper and lower sublayers or further extended along the original tubes. On the other hand, the $k$-space plots of excitons shown in Fig. 10 give the detailed information of optical transitions. The first exciton arises from the electron-hole excitation located mainly at the $X$ point, inclusively from the first valence band (v1) to the first conduction band (c1). Similar excitation is found for the second exciton, but a strong involvement of the transitions from $\mathrm{v} 2$ to $\mathrm{c} 1$ as well as $10 \%$ from $\mathrm{v} 3$ to $\mathrm{c} 1$ are identified. For the third excitation, most transitions are from $\mathrm{v} 1$ to $\mathrm{c} 1$. The fourth exciton, the fifth exciton, as well as the sixth exciton have similar transitions from v2 to $\mathrm{c} 1$, v3 to $\mathrm{c} 1$, and $\mathrm{v} 1$ to $\mathrm{c} 2$. The electron-hole excitations in Hittorf's phosphorene are not as highly localized in $k$ space as that in the $\mathrm{MoS}_{2}$ monolayer [5]. As we mentioned before, the less dispersive band structure associated with the complicated crystal structure in Hittorf's phosphorene invokes more electron-hole pairs in the BZ in forming excitons. With mixing the transitions between different electronic states, the optical selection rule could give rise to a certain kind of anisotropy in 2D materials [60]. The exact polarization angle dependence of optical absorption is shown in Fig. 11 for these first six exciton states.

Luminescence is another important aspect for the applications of a semiconductor. It is noted that wide-band-gap semiconductors such as GaN, AlN, and their alloys play an important role in the application of green and blue LED technology [61]. To access relevant properties in this $2 \mathrm{D}$ wideband-gap semiconductor, we further calculate the lifetime of the first bright exciton. Using Fermi's "golden rule," the radiative lifetime $\tau_{\mathrm{S}}(0)$ at $0 \mathrm{~K}$ of an exciton in state $S$ is derived according to $[10,62]$

$$
\tau_{S}(0)=\frac{\hbar^{2} c}{4 \pi e^{2} E_{\mathrm{S}}(0)} \frac{A_{\mathrm{uc}}}{\mu_{\mathrm{S}}^{2}},
$$

where $c$ is the speed of light, $A_{\mathrm{uc}}$ is the area of the unit cell, $E_{\mathrm{S}}(0)$ is the energy of the exciton in state $S$, and $\mu_{\mathrm{S}}^{2}=$ $\left[\hbar^{2} / m^{2} E_{\mathrm{S}}(0)^{2}\right]\left(\left|\left\langle G\left|p_{\|}\right| \Psi_{\mathrm{S}}\right\rangle\right|^{2} / N_{k}\right)$ is the square modulus of the BSE exciton transition dipole divided by the number of unit cells in this $2 \mathrm{D}$ system. We obtain the exciton radiative lifetime $\left\langle\tau_{\mathrm{S}}\right\rangle$ at temperature $T$ :

$$
\left\langle\tau_{\mathrm{S}}\right\rangle=\tau_{\mathrm{S}}(0) \frac{3}{4}\left(\frac{E_{\mathrm{S}}(0)^{2}}{2 M_{\mathrm{S}} c^{2}}\right)^{-1} k_{\mathrm{B}} T,
$$



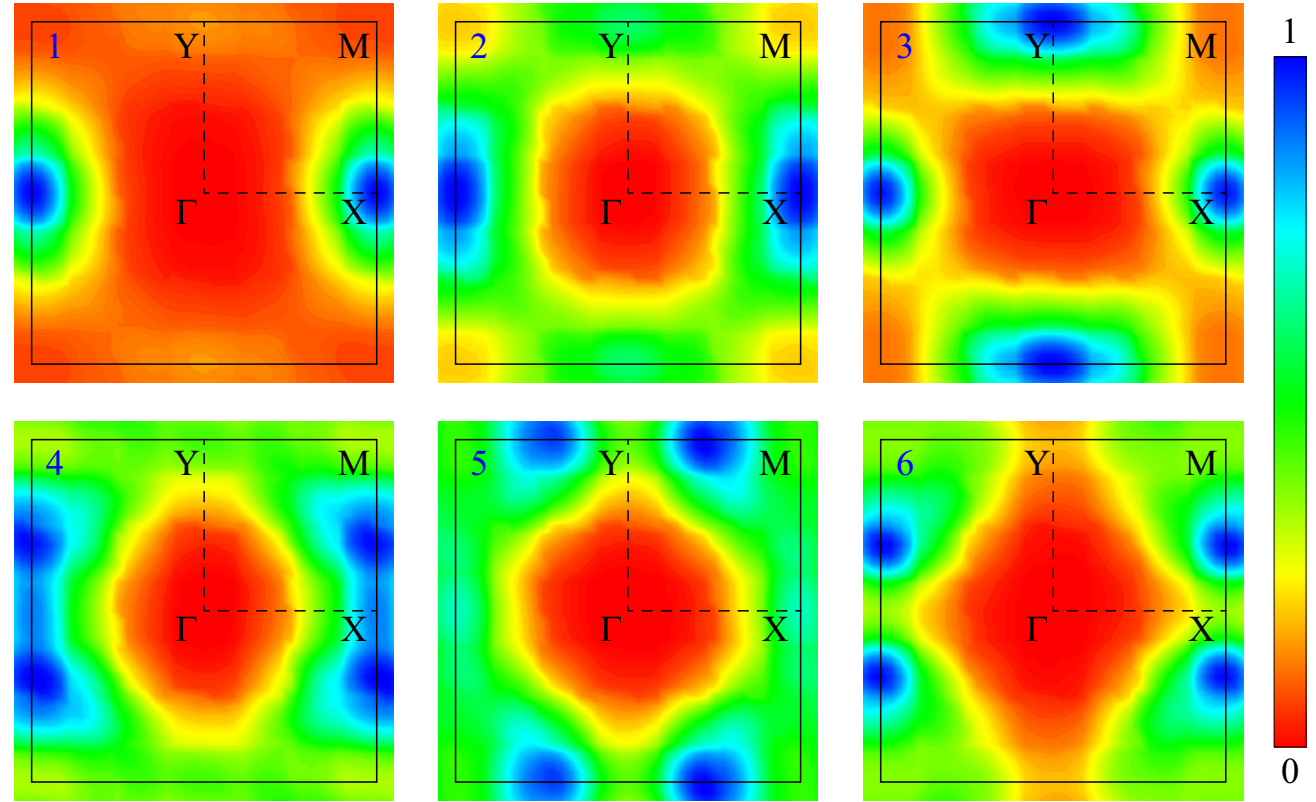

FIG. 10. $k$-space modulus squared of the wave function of the first six excitons in 2D Hittorf's phosphorene.

where $k_{\mathrm{B}}$ is Boltzmann constant and $M_{\mathrm{S}}=m_{e}^{*}+m_{h}^{*}$ is the exciton mass. Since the lowest bright exciton is arising from light polarized along the $x$ direction, the effective masses are fitted from the DFT band structure near band edge $X$ along the $\Gamma-X$ direction [62]. The computed radiative lifetime of the lowest-energy exciton at 0 and $4 \mathrm{~K}$ is 3 and $240 \mathrm{ps,}$ which is two orders of magnitude larger than the monolayer of transition-metal dichalcogenides, e.g., 4 ps in $\mathrm{MoS}_{2}$ at $4 \mathrm{~K}$ [10]. At room temperature ( $300 \mathrm{~K})$, the lifetime in Hittorf's phosphorene is around $18 \mathrm{~ns}$, which is of a similar order with the GaN single layer [23]. Compared with conventional wide-band-gap LED materials, e.g., the GaInN/GaN quantum well $(\mathrm{QW})$ with the radiative lifetime around $10^{2} \mathrm{~ns}$ [63], the relatively short lifetime in $2 \mathrm{D}$ materials shows its advantage in
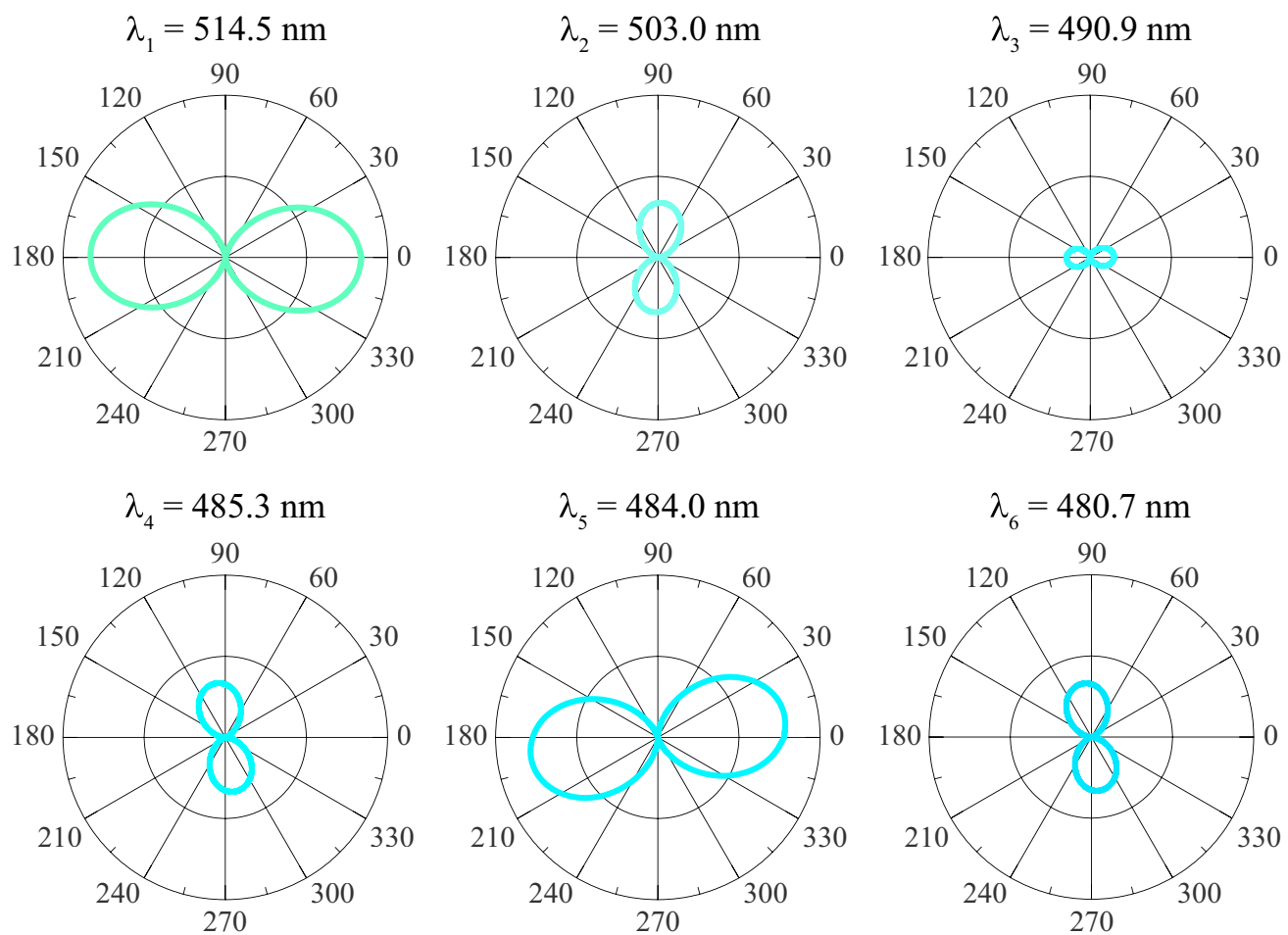

FIG. 11. Optical anisotropy (absorbance amplitude) for the first six excitonic states with the wavelength of 514.5, 503.0, 490.9, 485.3, 484.0 , and $480.7 \mathrm{~nm}$, respectively. 
TABLE II. Collection of exciton radiative lifetime $\tau(\mathrm{ns})$ for various $2 \mathrm{D}$ materials. $\tau_{\mathrm{S}}^{\mathrm{LT}}$ and $\tau_{\mathrm{S}}^{\mathrm{RT}}$ are the computed radiative lifetimes at low temperature $(\approx 4 \mathrm{~K})$ and room temperature, respectively. Experimental data of $\tau_{\exp }^{\mathrm{LT}}$ and $\tau_{\exp }^{\mathrm{RT}}$ are also listed for comparison.

\begin{tabular}{|c|c|c|c|c|}
\hline & $\tau_{\mathrm{S}}^{\mathrm{LT}}$ & $\tau_{\exp }^{\mathrm{LT}}$ & $\tau_{\mathrm{S}}^{\mathrm{RT}}$ & $\tau_{\exp }^{\mathrm{RT}}$ \\
\hline Hittorf's phosphorus & 0.24 & & 18 & \\
\hline Black phosphorus & & & $0.002[64]$ & \\
\hline $\mathrm{GeS}$ & & & $0.005[64]$ & \\
\hline $\mathrm{MoS}_{2}$ & $0.004[10]$ & $0.005[65], 0.005[66]$ & $0.27[10]$ & $0.85[67]$ \\
\hline $\mathrm{MoSe}_{2}$ & $0.005[10]$ & $0.002[68]$ & $0.38[10]$ & $0.9[69]$ \\
\hline $\mathrm{WS}_{2}$ & $0.002[10]$ & & $0.17[10]$ & \\
\hline $\mathrm{WSe}_{2}$ & $0.004[10]$ & $0.002[68], 0.004[70]$ & $0.29[10]$ & $4[71]$ \\
\hline $\mathrm{GaN}$ & & & $0.6[23]$ & \\
\hline GaInN/GaN QW & & & & $10^{2}[63]$ \\
\hline
\end{tabular}

LED applications. In Table II, we also list the exciton lifetime of several 2D materials, where all of them show relatively short lifetime [64-71]. On the other hand, besides radiative lifetime, the high mobility revealed in Hittorf's phosphorene is also beneficial for LED techniques [40].

With the above analysis, it is interesting to expect the potential applications of Hittorf's phosphorene in 2D optoelectronics to be similar to those for transition-metal dichalcogenides [7-9]. As shown in Fig. 12, we have plotted a schematic illustration of a $2 \mathrm{D}$ optoelectronic device based on Hittorf's phosphorene with split gate electrodes. Two gate electrodes couple to different regions of the layer. First, biasing one gate with a positive voltage $\left(V_{G 1}\right)$ and the other with a negative voltage $\left(V_{G 2}\right)$ will draw electrons and holes, respectively, and thus a $p-n$ junction is formed. By using a source-drain voltage $\left(V_{D S}\right)$, the electrons and holes transporting in antiparallel directions can recombine in the junction and emit photons, making the device operate as a LED. Similarly, when operating as a solar cell or photodiode, with the incident light absorbed in this $p-n$ junction, the photocurrent and photovoltage will be generated. Such a $2 \mathrm{D}$ monolayer $p$ - $n$ junction is very effective in a photovoltaic solar cell, photodiode, and LED, overcoming many limitations (e.g., rigidity, heavy weight, and high costs) in the conventional bulk semiconductors. Here, for the light emitting, extrinsic factors such as $V_{G 1}$ and $V_{G 2}$ and intrinsic properties such as exciton lifetime and carrier mobility determine the ultimate quantum efficiency of 2D Hittorf's phosphorene. For the photovoltaics, the strong absorbance in the visible light range and sufficient lifetime for electron-hole separation (compared with the $\mathrm{MoS}_{2}$ monolayer [10]) suggest that the system could be effective when acting as a solar cell. For the polarized light, as shown in Fig. 11, the significant anisotropy associated with absorbance amplitude is obvious for each wavelength of optical excitations. At higher energies, although exciton states become dense, the difference of absorbance between two directions is still obvious, indicating the potential application as an optical polarizer.

Finally, we should mention that the defect [72,73] could change the optical spectrum in Hittorf's phosphorene strongly (see Appendix F). The defect formation energy is similar to that in transition-metal dichalcogenides [74] and black phosphorus [75]. In the meantime, similar to black phosphorus [6], when increasing the layer number, the systems of Hittorf's phosphorus show a reduced band gap (see Appendix $\mathrm{G})$. The multilayer system as well as bulk are indirect-bandgap semiconductors. These findings indicate that the single layer of Hittorf's phosphorene with a direct band gap and unusual optical properties could play an important role in optoelectronics.

\section{SUMMARY}

To summarize, based on DFT with $G W$-BSE method, we have studied the quasiparticle electronic structure, exciton, and optical spectrum of 2D Hittorf's phosphorene. The direct band gap of $3.32 \mathrm{eV}$ and first excitonic state at $2.41 \mathrm{eV}$ bridge the gap between transition-metal dichalcogenides and III-VI

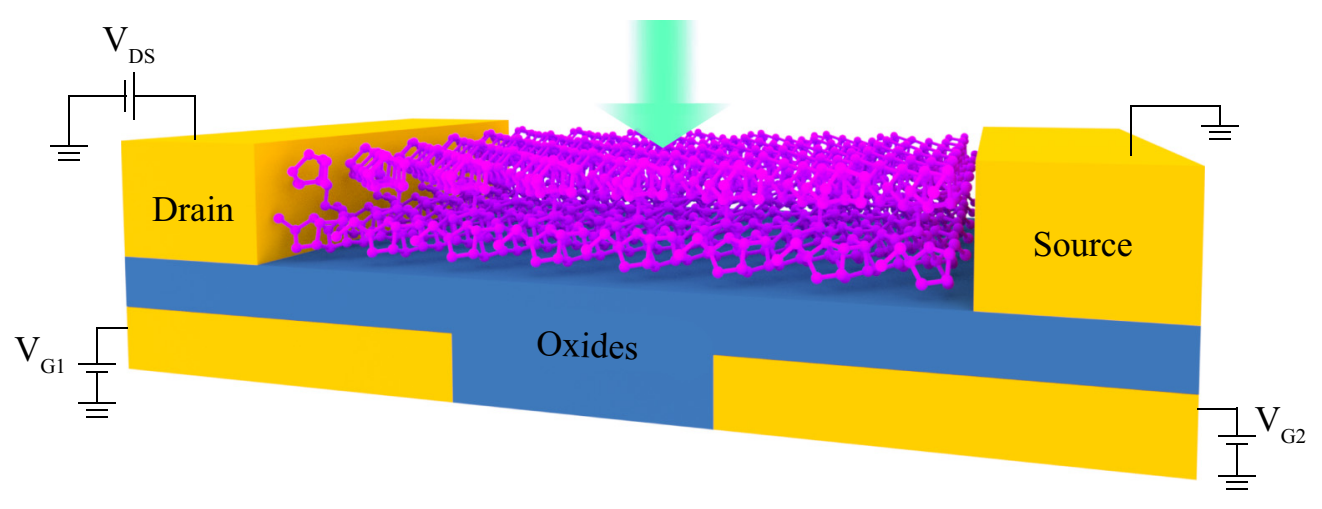

FIG. 12. Illustration of the optoelectronic diode based on 2D Hittorf's phosphorene. 
(a)

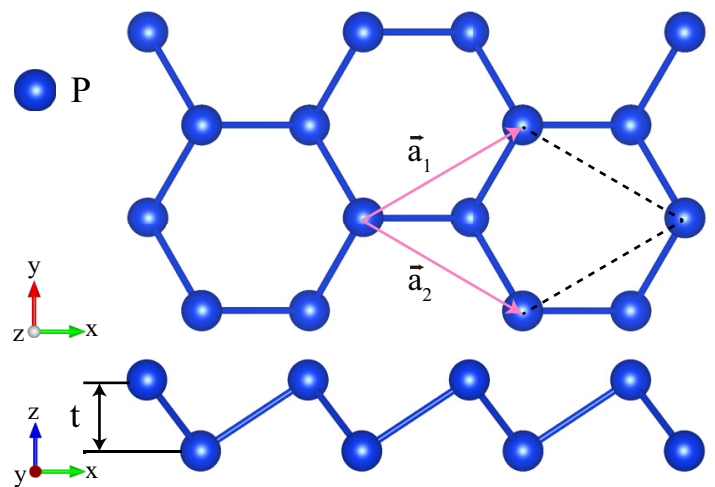

(b)
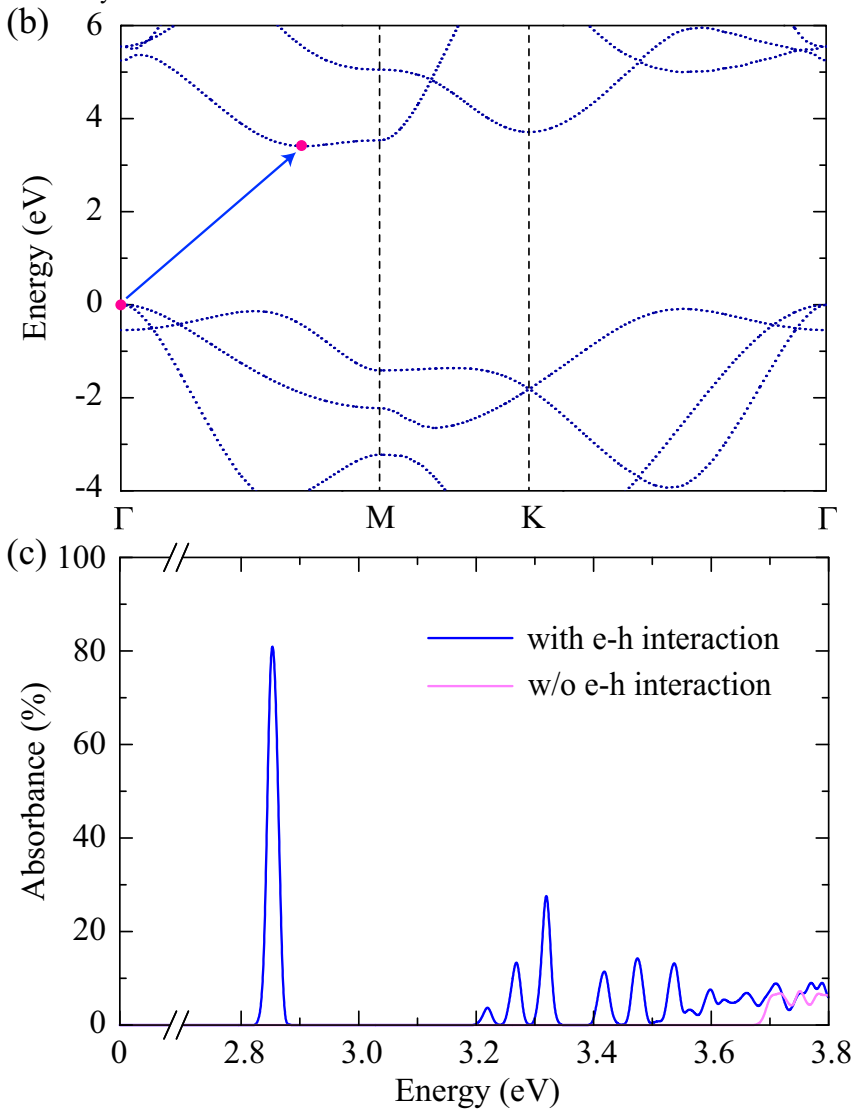

FIG. 13. (a) Crystal structure of 2D blue phosphorene with inplane lattice constant $(a)$ of $3.28 \AA$ and effective thickness $(t)$ of $1.22 \AA$. (b) Quasiparticle band structure of 2D blue phosphorene with an indirect band gap of $3.41 \mathrm{eV}$ indicated by an arrow. (c) Optical absorbance of 2D blue phosphorene with absorption edge located at $2.85 \mathrm{eV}$ arising from the first exciton state. Here, the calculations are performed with a high accuracy in a $30 \times 30 \times 1$ coarse $k$ grid, 500 total bands, and dielectric cutoff of 10 Ry for quasiparticle band energy. The optical absorption is performed in a $120 \times 120 \times 1 k$ grid.

monochalcogenides in the optical spectrum of 2D materials. The relatively short lifetime of the lowest exciton and high carrier mobility promise a good chance to realize a green light LED. Furthermore, the anisotropic electron-hole excitations over a wide spectral range give rise to obvious polarization direction dependence of optical absorbance. Our findings have provided strong evidences for the applications of Hittorf's phosphorene in optoelectronics.
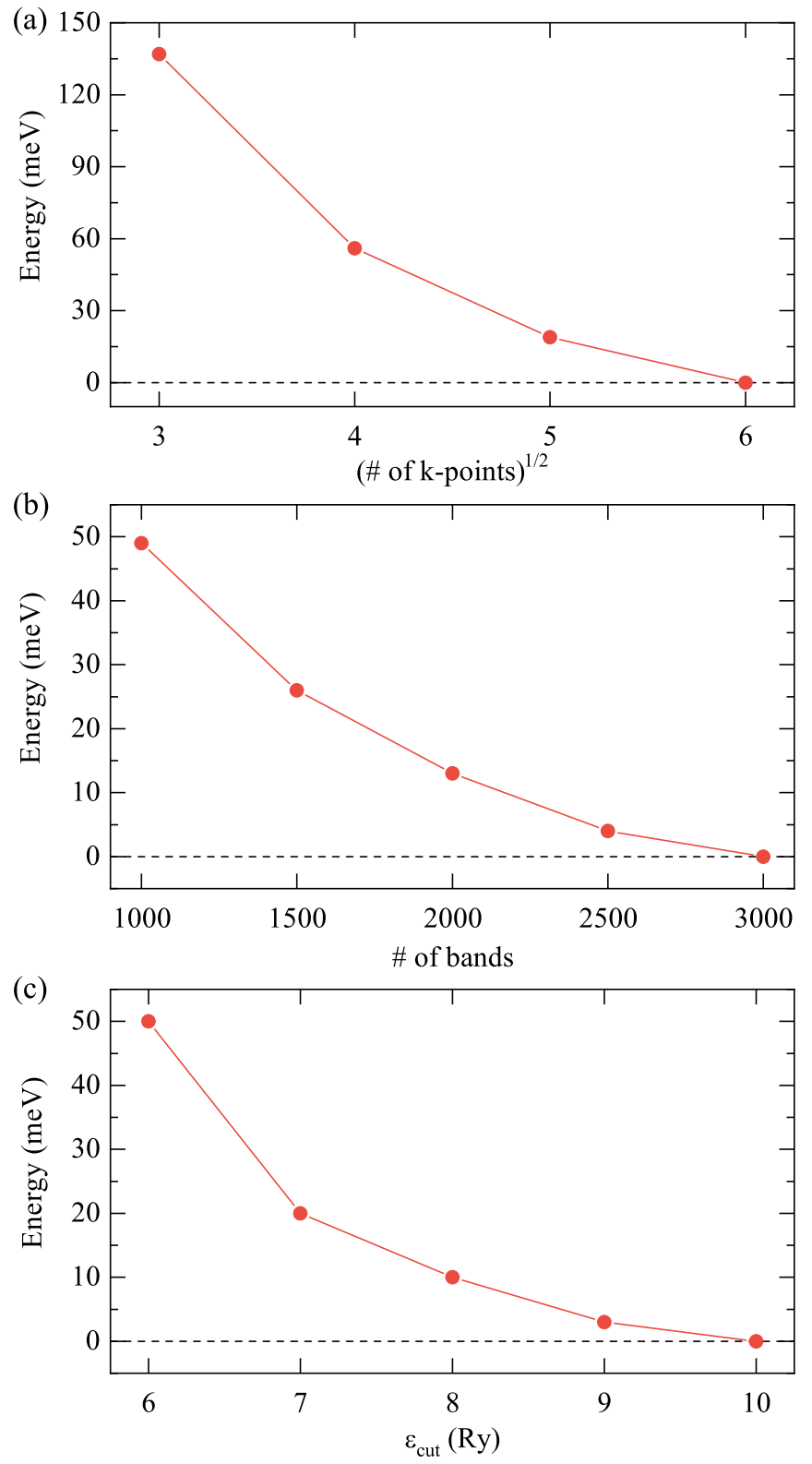

FIG. 14. Convergence of the quasiparticle band gap of 2D Hittorf's phosphorene on (a) the scale of a coarse $k$ grid (here, the number of bands is 3150 and the dielectric cutoff is $10 \mathrm{Ry}$ ), (b) the number of total bands (here, the coarse $k$ grid is $6 \times 6 \times 1$ and the dielectric cutoff is $10 \mathrm{Ry}$ ), and (c) the dielectric cutoff (here, the coarse $k$ grid is $6 \times 6 \times 1$ and the number of bands is 3150 ).

\section{ACKNOWLEDGMENTS}

S.J. appreciates Prof. Steven G. Louie and all his group members for their great help on BERKELEYGW method and useful discussion on exciton physics in 2D materials. J.Z. thanks Prof. A. S. Rodin for the helpful discussion on the model calculation of binding energy in 2D materials. This work is supported by the National Basic Research Program of China (973 Program No. 2019YFA0308402) and National Natural Science Foundation of China under Grant No. 51972217. 
TABLE III. Optimized atomic positions (fractional coordinates in terms of the lattice constants) for 2D Hittorf's phosphorene with the lattice constants $a=9.24(\AA), b=9.26(\AA)$, and $c=30.16(\AA)$. For Hittorf's bulk, the optimized crystal structure [with $a=9.24(\AA), b=$ $9.16(\AA), c=22.57(\AA)$, and $\beta=106.1^{\circ}$ ] is in good agreement with experimental data [38].

\begin{tabular}{|c|c|c|c|c|c|c|c|}
\hline Atom & $x$ & $y$ & $z$ & Atom & $x$ & $y$ & $z$ \\
\hline $\mathrm{P} 1$ & 0.358966 & 0.339682 & 0.333279 & $\mathrm{P} 22$ & 0.672558 & 0.975614 & 0.535874 \\
\hline $\mathrm{P} 2$ & 0.589423 & 0.619228 & 0.344934 & $\mathrm{P} 23$ & 0.788657 & 0.341854 & 0.554517 \\
\hline P3 & 0.078462 & 0.110784 & 0.345280 & $\mathrm{P} 24$ & 0.042184 & 0.090717 & 0.554767 \\
\hline P4 & 0.828000 & 0.872082 & 0.347673 & $\mathrm{P} 25$ & 0.891170 & 0.905699 & 0.558140 \\
\hline P5 & 0.358960 & 0.567229 & 0.356215 & $\mathrm{P} 26$ & 0.603248 & 0.192456 & 0.559183 \\
\hline P6 & 0.131208 & 0.340541 & 0.356463 & $\mathrm{P} 27$ & 0.309528 & 0.836080 & 0.562379 \\
\hline P7 & 0.598173 & 0.845290 & 0.368081 & $\mathrm{P} 28$ & 0.535737 & 0.610239 & 0.562694 \\
\hline P8 & 0.852486 & 0.101046 & 0.368999 & P29 & 0.166072 & 0.642646 & 0.570288 \\
\hline P9 & 0.480191 & 0.218842 & 0.388956 & P30 & 0.341629 & 0.467415 & 0.570458 \\
\hline P10 & 0.207324 & 0.989830 & 0.398552 & P31 & 0.955498 & 0.256524 & 0.600957 \\
\hline P11 & 0.710582 & 0.492219 & 0.398650 & $\mathrm{P} 32$ & 0.710581 & 0.507780 & 0.601349 \\
\hline P12 & 0.955498 & 0.743475 & 0.399042 & P33 & 0.207324 & 0.010169 & 0.601447 \\
\hline P13 & 0.341629 & 0.532584 & 0.429541 & P34 & 0.480191 & 0.781157 & 0.611043 \\
\hline P14 & 0.166072 & 0.357352 & 0.429711 & P35 & 0.852486 & 0.898953 & 0.631000 \\
\hline P15 & 0.535737 & 0.389760 & 0.437305 & P36 & 0.598173 & 0.154710 & 0.631918 \\
\hline P16 & 0.309528 & 0.163919 & 0.437620 & P37 & 0.131208 & 0.659458 & 0.643536 \\
\hline P17 & 0.603248 & 0.807543 & 0.440816 & P38 & 0.358960 & 0.432770 & 0.643784 \\
\hline P18 & 0.891170 & 0.094300 & 0.441859 & P39 & 0.828000 & 0.127917 & 0.652326 \\
\hline P19 & 0.042184 & 0.909282 & 0.445232 & P40 & 0.078462 & 0.889215 & 0.654719 \\
\hline P20 & 0.788657 & 0.658145 & 0.445482 & $\mathrm{P} 41$ & 0.589423 & 0.380771 & 0.655065 \\
\hline $\mathrm{P} 21$ & 0.672558 & 0.024385 & 0.464125 & $\mathrm{P} 42$ & 0.358966 & 0.660317 & 0.666720 \\
\hline
\end{tabular}

\section{APPENDIX A: QUASIPARTICLE BAND STRUCTURE AND OPTICAL ABSORPTION OF BLUE PHOSPHORENE}

Blue phosphorene, a 2D phosphorus honeycomb structure as shown in Fig. 13(a), was first proposed by Zhu and Tománek [29]. The band gap within Perdew-Burke-Ernzerhof (PBE) is around $2.0 \mathrm{eV}$. Here, as shown in Fig. 13(b), this $2 \mathrm{D}$ system is an indirect-band-gap semiconductor. The top of the valence band is located at the $\Gamma$ point. The bottom of the conduction band is located at the $k$ point along $\Gamma-M$. The quasiparticle band gap within $G_{0} W_{0}$ is $3.41 \mathrm{eV}$. From Fig. 13(c), the optical responses in this $2 \mathrm{D}$ system are dominated by excitonic effect. The first exciton state at $2.85 \mathrm{eV}$ is bright with a large binding energy of $0.84 \mathrm{eV}$. The magnitude of binding energy is similar to the $\mathrm{MoS}_{2}$ monolayer [5]. The
$G W$-BSE calculations for blue phosphorene are performed with the BERKELEYGW package [55]. The accuracy for both quasiparticle energies and exciton states is within $0.1 \mathrm{eV}$. These calculations are also in good agreement with previous results [30] based on the BERKELEYGW package.

\section{APPENDIX B: ATOMIC STRUCTURE OF HITTORF'S PHOSPHORENE AND THE PHOSPHORUS NANOTUBE}

The crystal structure of Hittorf's phosphorene is fully optimized. In Table III, we show the exact atomic position as well as lattice constants of 2D Hittorf's phosphorene in our simulations. In Table IV, we show the optimized atomic position as well as lattice constants of the phosphorus nanotube in our simulations.

TABLE IV. Optimized atomic positions (fractional coordinates in terms of the lattice constants) for the 1D phosphorus nanotube with the lattice constants $a=20.00(\AA), b=20.00(\AA)$, and $c=12.98(\AA)$. Here, an H atom is added to passivate the dangling bond when a tube is extracted from the 2D system.

\begin{tabular}{|c|c|c|c|c|c|c|c|}
\hline Atom & $x$ & $y$ & $z$ & Atom & $x$ & $y$ & $z$ \\
\hline $\mathrm{P} 1$ & 0.052382 & 0.035871 & -0.433460 & $\mathrm{P} 12$ & -0.020010 & 0.126970 & 0.063453 \\
\hline $\mathrm{P} 2$ & 0.060115 & 0.047987 & -0.687060 & P13 & 0.110712 & 0.181656 & -0.524740 \\
\hline P3 & 0.061981 & 0.048465 & -0.186290 & P14 & 0.111343 & 0.183914 & -0.347690 \\
\hline P4 & 0.061775 & 0.048823 & 0.063644 & P15 & 0.116916 & 0.186943 & 0.208938 \\
\hline P5 & 0.122370 & 0.071839 & -0.551360 & P16 & 0.000168 & 0.186998 & -0.550940 \\
\hline P6 & 0.124457 & 0.074282 & -0.319090 & P17 & 0.116286 & 0.188442 & -0.078590 \\
\hline P7 & 0.129427 & 0.078658 & 0.191284 & P18 & 0.000766 & 0.188691 & -0.322460 \\
\hline P8 & 0.129922 & 0.079754 & -0.061370 & P19 & 0.006581 & 0.195802 & -0.062450 \\
\hline P9 & -0.031980 & 0.112932 & -0.435420 & $\mathrm{P} 20$ & 0.007747 & 0.197018 & 0.189336 \\
\hline P10 & -0.020760 & 0.126308 & -0.187030 & $\mathrm{P} 21$ & 0.166711 & 0.217787 & 0.066137 \\
\hline P11 & -0.021880 & 0.126721 & -0.688890 & $\mathrm{H}$ & 0.158009 & 0.287713 & 0.079542 \\
\hline
\end{tabular}




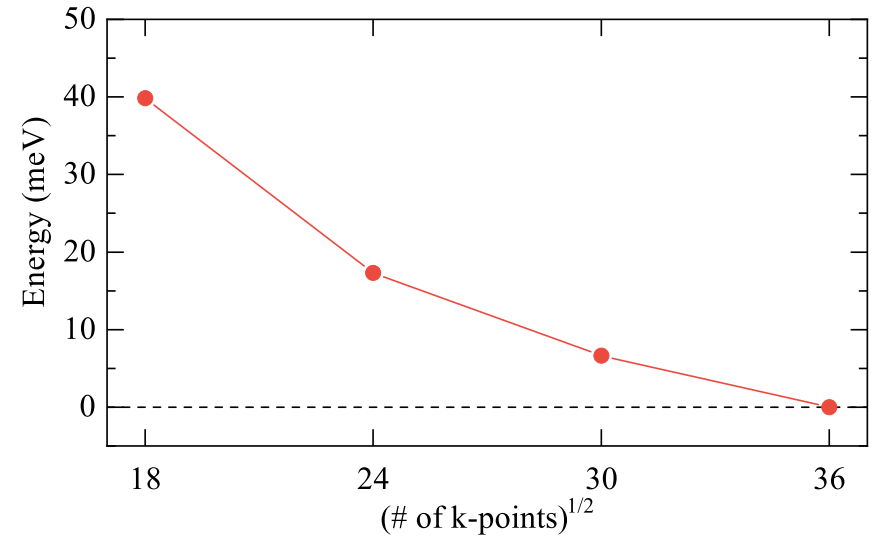

FIG. 15. Convergence of the calculated first exciton energy in 2D Hittorf's phosphorene on the scale of a fine $k$ grid.

\section{APPENDIX C: CONVERGENCE OF $G W$-BSE CALCULATIONS OF HITTORF'S PHOSPHORENE}

In spite of a complicated crystal structure with 42 atoms in the unit cell, the convergence of $G_{0} W_{0}$-BSE results of Hittorf's phosphorene is carefully performed within our computational limit. Figures 14 and 15 are for the convergence of $G_{0} W_{0}$ and BSE, respectively. The accuracy of current calculations is within $0.1 \mathrm{eV}$.

\section{APPENDIX D: MODEL OF EXCITON BINDING ENERGY IN 2D MATERIALS}

Here, we use the 2D exciton model [49] for the exciton binding energy in Hittorf's phosphorene. We consider the effective mass approximation for the exciton states, where the 2D Schrödinger Hamiltonian for an anisotropic system can be expressed as

$$
H=-\frac{\hbar^{2}}{4 \bar{\mu}} \nabla^{2}-V\left(\frac{r \sqrt{1+\beta \cos 2 \phi}}{r_{0}}\right) .
$$

Here,

$$
\bar{\mu}=\frac{\mu^{x} \mu^{y}}{\mu^{x}+\mu^{y}}
$$

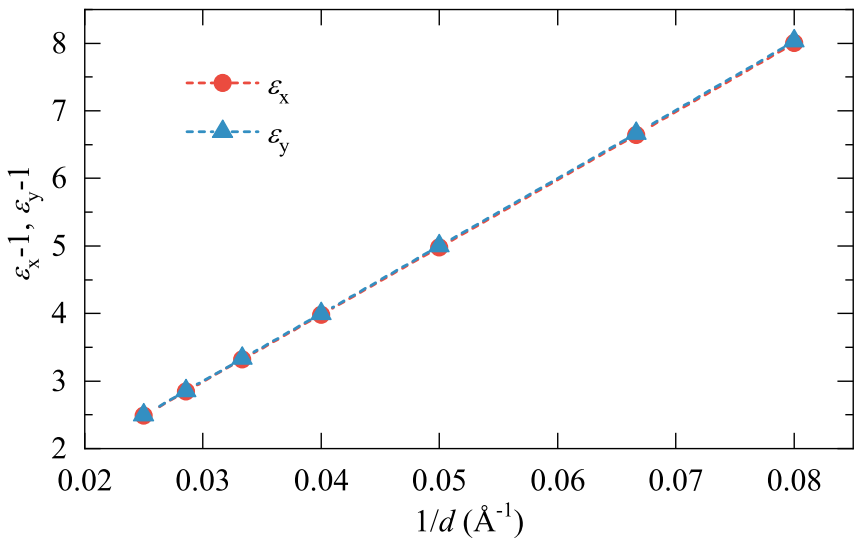

FIG. 16. In-plane dielectric constant $\varepsilon_{x, y}$ of Hittorf's phosphorene as a function of the inverse interlayer distance $1 / d$.
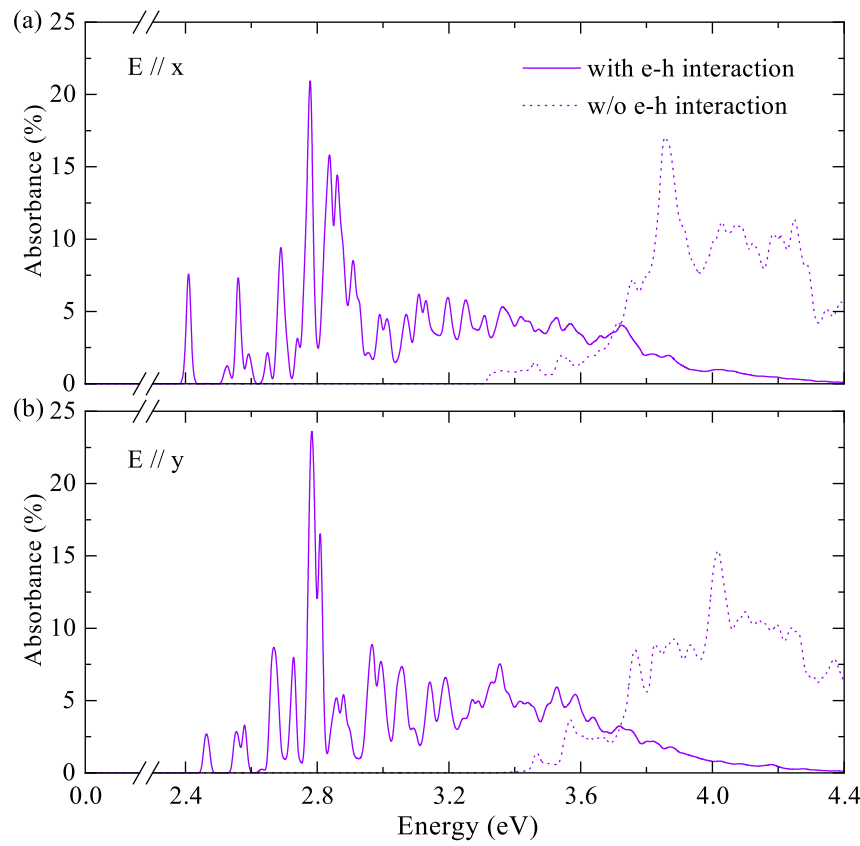

FIG. 17. Optical absorbance of a 2D Hittorf's phosphorus monolayer with and without the inclusion of electron-hole interaction. (a) Electric polarization along the $x$ axis and (b) electric polarization along the $y$ axis.

is the reduced mass for excitons (electron-hole pairs) with

$$
\mu^{x / y}=\frac{m_{e}^{x / y} m_{h}^{x / y}}{m_{e}^{x / y}+m_{h}^{x / y}},
$$

where $\mu^{x / y}$ is the reduced mass in specific directions $(x / y)$ with effective masses of the electron $\left(m_{e}^{x / y}\right)$ and the hole $\left(m_{h}^{x / y}\right)$. We use $\beta=\left|\left(\mu^{y}-\mu^{x}\right) /\left(\mu^{y}+\mu^{x}\right)\right|$ as the anisotropic parameter. $r_{0}=2 \pi \alpha_{2 \mathrm{D}}$ is the length scale of $2 \mathrm{D}$ dielectric screening and $\alpha_{2 \mathrm{D}}$ is the 2D polarizability.

To calculate the 2D polarizability of Hittorf's phosphorene, we use the relation between the static dielectric constant $\varepsilon$ and

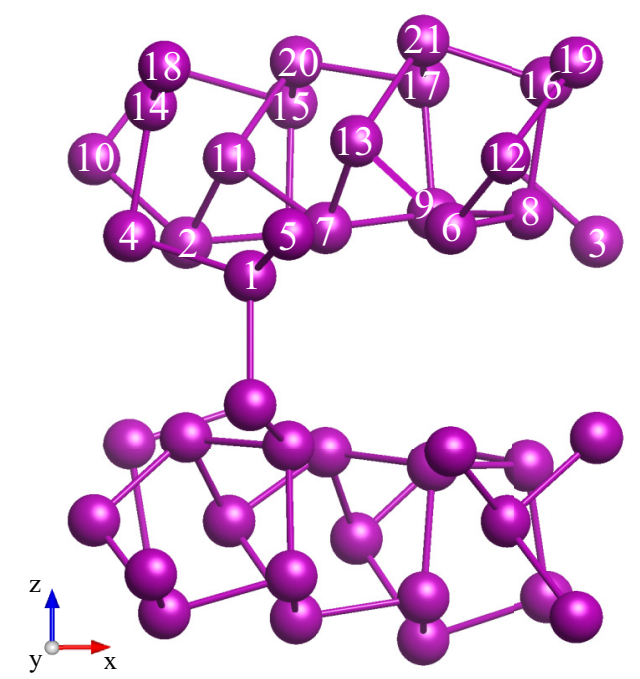

FIG. 18. Hittorf's phosphorene crystal structure with the number indicating the site of vacancy. 

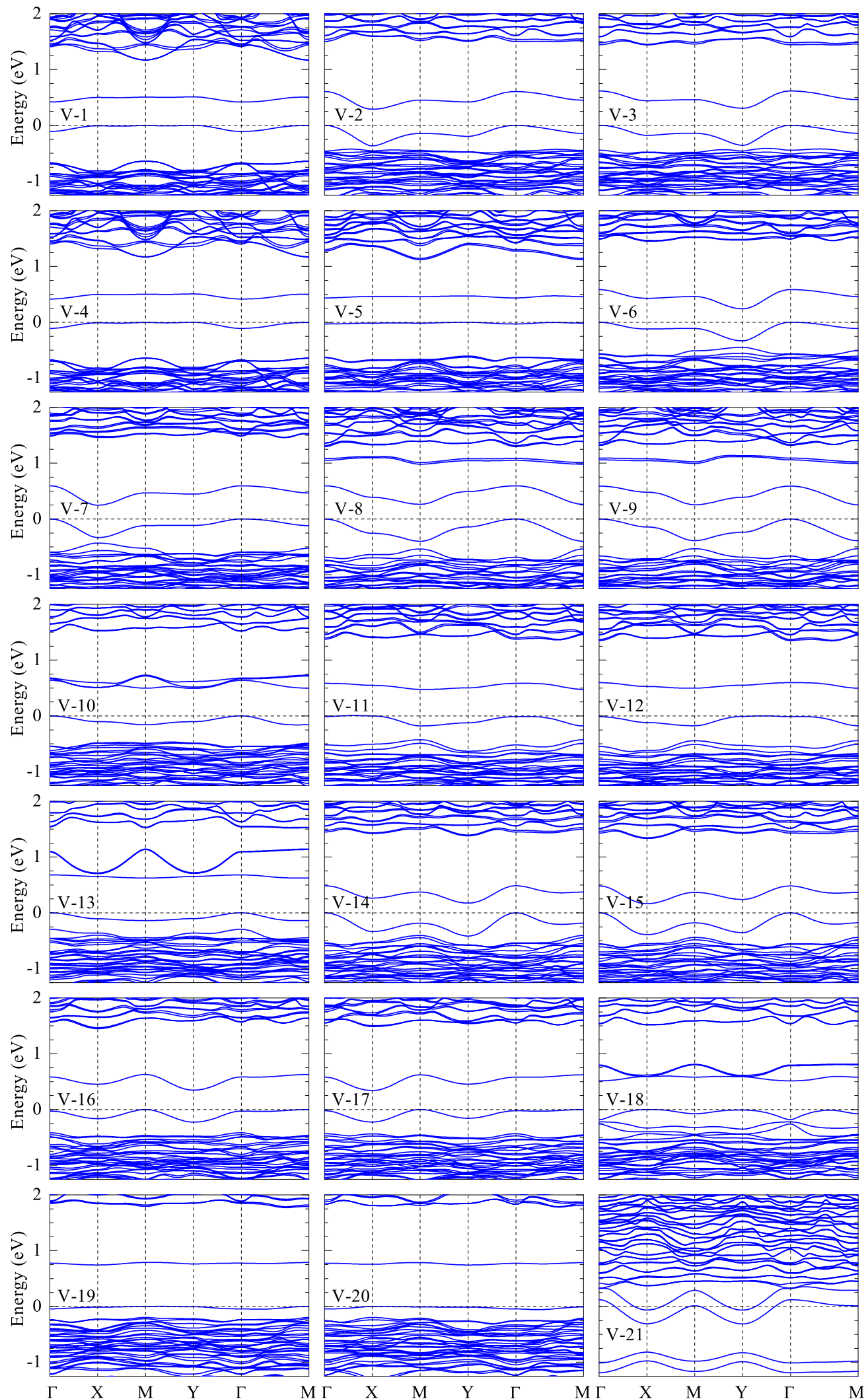

FIG. 19. Electronic band structures (PBE level) of 2D Hittorf's phosphorene for different phosphorus monovacancy sites.

the interlayer distance $d$ [49]:

$$
\varepsilon_{x, y}(d)=1+\frac{4 \pi \alpha_{x x, y y}}{d}
$$

The results are shown in Fig. 16, with a linear dependence between $\varepsilon_{x, y}$ and the inverse interlayer distance $1 / d$. According to Eq. (D4), we get $\alpha_{x x}=7.95 \AA$ and $\alpha_{y y}=7.96 \AA$ and we use the average value $\alpha=7.95 \AA$. This yields the screening 
TABLE V. The defect formation energy $E^{f}$ of phosphorus vacancy at different sites.

\begin{tabular}{lccccc}
\hline \hline Site & $E^{f}(\mathrm{eV})$ & Site & $E^{f}(\mathrm{eV})$ & Site & $E^{f}(\mathrm{eV})$ \\
\hline V1 & 1.05 & $\mathrm{~V} 8$ & 2.20 & $\mathrm{~V} 15$ & 1.62 \\
V2 & 1.67 & $\mathrm{~V} 9$ & 2.21 & $\mathrm{~V} 16$ & 1.59 \\
V3 & 1.67 & $\mathrm{~V} 10$ & 2.61 & $\mathrm{~V} 17$ & 1.59 \\
V4 & 1.05 & $\mathrm{~V} 11$ & 1.60 & $\mathrm{~V} 18$ & 2.63 \\
V5 & 1.30 & $\mathrm{~V} 12$ & 1.59 & $\mathrm{~V} 19$ & 1.49 \\
V6 & 1.55 & $\mathrm{~V} 13$ & 2.44 & $\mathrm{~V} 20$ & 1.53 \\
V7 & 1.56 & $\mathrm{~V} 14$ & 1.60 & $\mathrm{~V} 21$ & 2.35 \\
\hline \hline
\end{tabular}

length $r_{0}=49.95 \AA$. For the effective masses, which are fitted from the band structure (PBE level), we get $m_{e}^{x}=0.65 m_{0}$ and $m_{e}^{y}=3.85 m_{0}$ for the electron and $m_{h}^{x}=1.15 m_{0}$ and $m_{h}^{y}=2.44$ $m_{0}$ for the hole. Using these values, we get the anisotropic parameter $\beta=0.56$ and the reduced mass $\bar{\mu}=0.32 \mathrm{~m}_{0}$.

To solve the Hamiltonian, we adopt an approximated expression of the screened Coulomb potential [49]:

$$
V(r)=-\frac{1}{r_{0}}\left[\ln \left(\frac{r}{r+r_{0}}\right)+(\gamma-\ln 2) e^{-\frac{r}{r_{0}}}\right],
$$

where $\gamma \approx 0.5772$ is Euler's constant. And the eigenfunctions are expressed as

$$
\Psi_{n}(r, \phi)=\sum_{l} f_{l}(r) \operatorname{trig}^{ \pm}(l \phi),
$$

where $l$ runs over the appropriate harmonic numbers for the basis function and trig ${ }^{ \pm}$is cosine or sine, respectively.

Then, the Hamiltonian in Eq. (D1) is solved using Numerov method and the exciton binding energy is determined by the bisection method. Finally, we get the exciton binding energy $E_{b}=0.87 \mathrm{eV}$ for the lowest-energy exciton of Hittorf's phosphorene, which is similar to the value of $0.91 \mathrm{eV}$ we obtained using the $G_{0} W_{0}$-BSE approach.
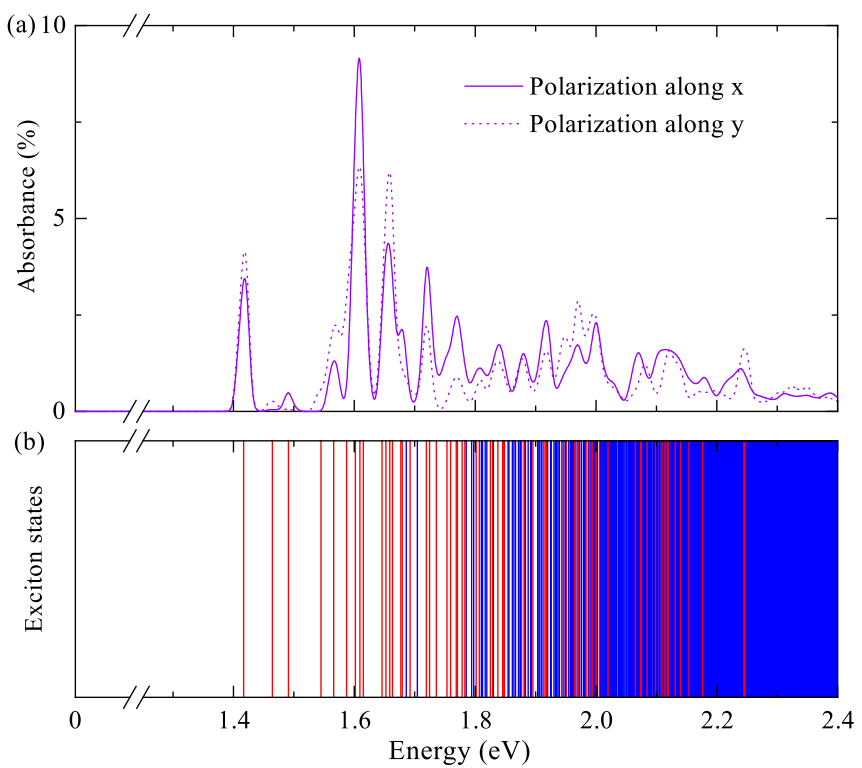

FIG. 20. $G_{0} W_{0}$-BSE approach of (a) optical absorbance and (b) exciton states (red line for bright exciton and blue line for dark exciton) of Hittorf's phosphorene with a defect of V1.

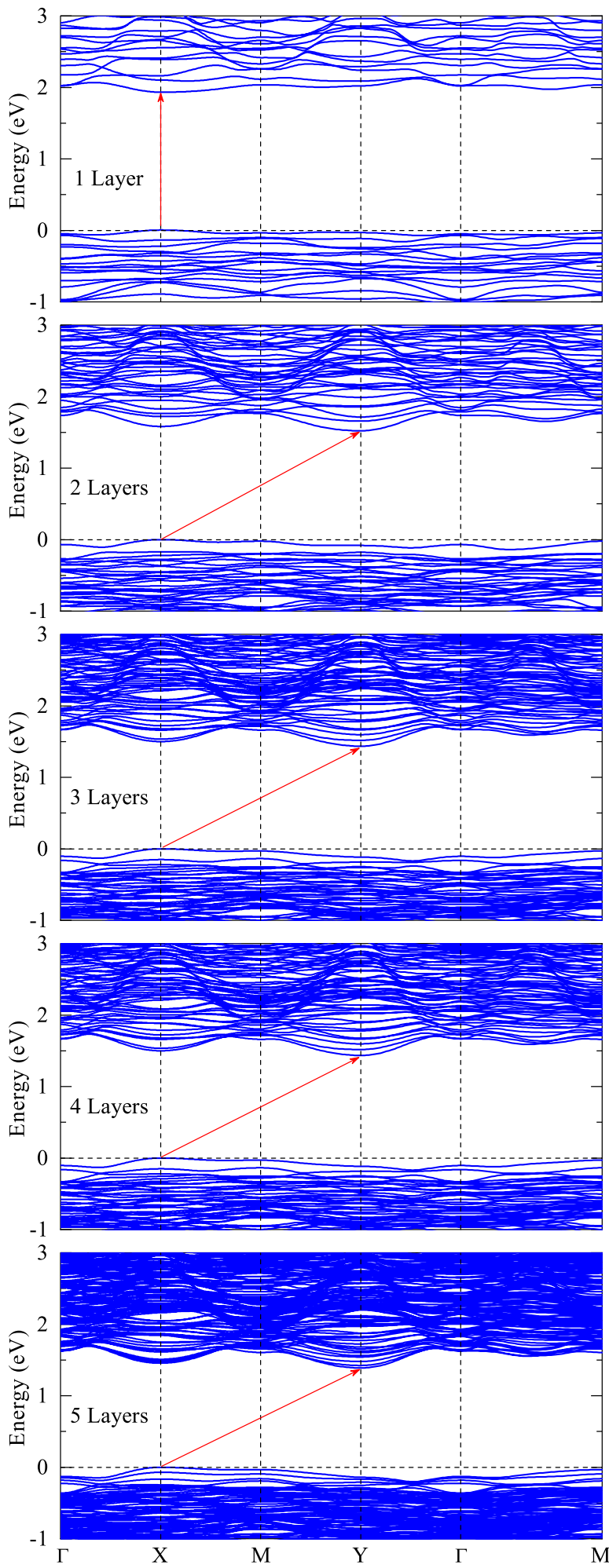

FIG. 21. Electronic band structures of few-layer Hittorf's phosphorus.

\section{APPENDIX E: ANISOTROPIC OPTICAL ABSORPTION IN HITTORF'S PHOSPHORENE}

In Fig. 17, we have shown the optical absorbance of 2D Hittorf's phosphorene with and without the inclusion 


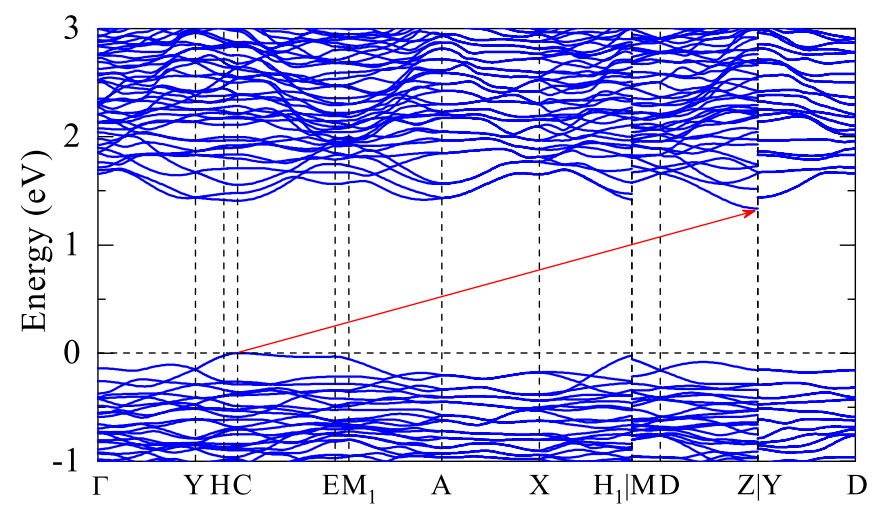

FIG. 22. Electronic band structures of bulk Hittorf's phosphorus.

of electron-hole interactions for both directions of electric polarizations.

\section{APPENDIX F: DEFECT IN HITTORF'S PHOSPHORENE}

The defect formation energy $E^{f}$ is defined as [72,73]

$$
E^{f}=E_{\text {defect }}-E_{\text {tot }}+n_{\mathrm{P}} \mu_{\mathrm{P}},
$$

where $E_{\text {defect }}$ is the total energy of the 2D Hittorf's phosphorene containing the phosphorus vacancy and $E_{\text {tot }}$ is the total energy of the pristine 2D Hittorf's phosphorene. $n_{\mathrm{P}}$ indicates the number of the phosphorus vacancy and $\mu_{\mathrm{P}}$ is the chemical potential of the phosphorus atoms. Here, the chemical potential of the phosphorus atom is obtained from the pristine 2D Hittorf's phosphorene [73]. In Table V, we show the formation energy of the 2D Hittorf's phosphorene with one of the phosphorus atoms removed. The number is indicated in Fig. 18 for 21 inequivalent sites. The lowest formation energy of around $1 \mathrm{eV}$ is found at V1 and V4 sites. The magnitude of formation energy is similar to $\mathrm{MoS}_{2}$ of $2.71 \mathrm{eV}$ [74] and black phosphorene of $1.96 \mathrm{eV}$ [75]. The electronic band structure at the PBE level is shown in Fig. 19. For its influence on the optical response in Hittorf's phosphorene, we provide the optical absorbance (within $G_{0} W_{0}$-BSE) in the system with

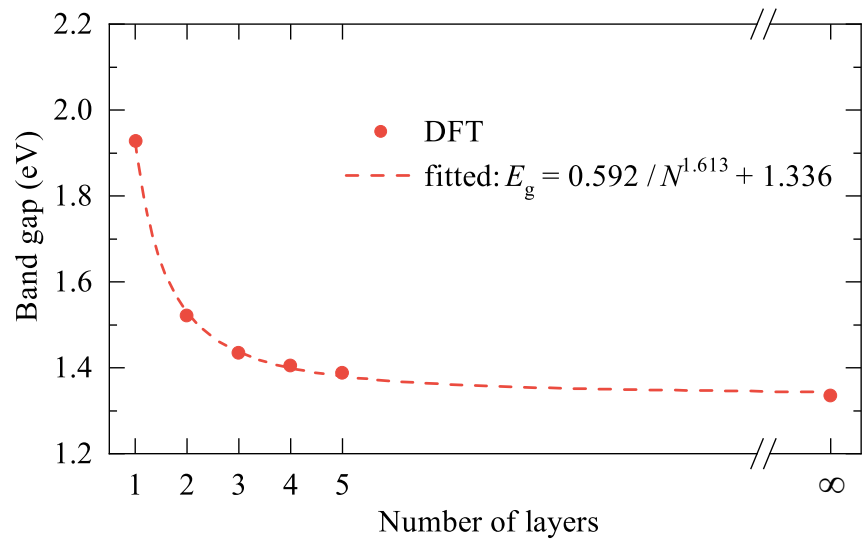

FIG. 23. Evolution of band gap $E_{g}$ with the layer number in Hittorf's phosphorus.

V1 (the most energetically favorable system) in Fig. 20(a). Clearly, compared with the pristine system, the absorption edge shows a redshift and optical anisotropy is reduced. The exciton states are also shown in Fig. 20(b).

\section{APPENDIX G: LAYER DEPENDENCE BAND STRUCTURE IN HITTORF'S PHOSPHORUS}

The electronic band structures (PBE level) of few-layer Hittorf's phosphorus are shown in Fig. 21. In Fig. 22, we show the band structure (PBE level) of the Hittorf's phosphorus bulk. In Fig. 23, we show the layer number $(N)$ dependence of band gap $E_{g}$ in Hittorf's phosphorus. Following the consideration in black phosphorus [13], we find the band gap (PBE level) decreases as a power law of $E_{g}=0.592 / N^{1.613}+1.336$. In addition, for few-layer Hittorf's phosphorus as well as the bulk, the systems are indirect-band-gap semiconductors. All these results indicate that Hittorf's phosphorene with a direct band gap could have potential applications in $2 \mathrm{D}$ optoelectronics.
[1] F. Xia, H. Wang, D. Xiao, M. Dubey, and A. Ramasubramaniam, Two-dimensional material nanophotonics, Nat. Photonics 8, 899 (2014).

[2] K. F. Mak and J. Shan, Photonics and optoelectronics of 2D semiconductor transition metal dichalcogenides, Nat. Photonics 10, 216 (2016).

[3] Z. Sun, A. Martinez, and F. Wang, Optical modulators with 2D layered materials, Nat. Photonics 10, 227 (2016).

[4] L. Yang, J. Deslippe, C. H. Park, M. L. Cohen, and S. G. Louie, Excitonic Effects on the Optical Response of Graphene and Bilayer Graphene, Phys. Rev. Lett. 103, 186802 (2009).

[5] D. Y. Qiu, F. H. da Jornada, and S. G. Louie, Optical Spectrum of $\mathrm{MoS}_{2}$ : Many-Body Effects and Diversity of Exciton States, Phys. Rev. Lett. 111, 216805 (2013); 115, 119901 (2015).

[6] R. Soklaski, Y. Liang, and L. Yang, Temperature effect on optical spectra of monolayer molybdenum disulfide, Appl. Phys. Lett. 104, 193110 (2014).
[7] A. Pospischil, M. M. Furchi, and T. Mueller, Solar-energy conversion and light emission in an atomic monolayer $p$ - $n$ diode, Nat. Nanotechnol. 9, 257 (2014).

[8] B. W. H. Baugher, H. O. H. Churchill, Y. Yang, and P. JarilloHerrero, Optoelectronic devices based on electrically tunable p-n diodes in a monolayer dichalcogenide, Nat. Nanotechnol. 9, 262 (2014).

[9] J. S. Ross, P. Klement, A. M. Jones, N. J. Ghimire, J. Yan, D. G. Mandrus, T. Taniguchi, K. Watanabe, K. Kitamura, W. Yao, D. H. Cobden, and $\mathrm{X}$. $\mathrm{Xu}$, Electrically tunable excitonic light-emitting diodes based on monolayer $\mathrm{WSe}_{2} p$ - $n$ junctions, Nat. Nanotechnol. 9, 268 (2014).

[10] M. Palummo, M. Bernardi, and J. C. Grossman, Exciton radiative lifetimes in two-dimensional transition metal dichalcogenides, Nano Lett. 15, 2794 (2015). 
[11] D. Y. Qiu, F. H. da Jornada, and S. G. Louie, Screening and many-body effects in two-dimensional crystals: Monolayer $\mathrm{MoS}_{2}$, Phys. Rev. B 93, 235435 (2016).

[12] W. Song and L. Yang, Quasiparticle band gaps and optical spectra of strained monolayer transition-metal dichalcogenides, Phys. Rev. B 96, 235441 (2017).

[13] V. Tran, R. Soklaski, Y. Liang, and L. Yang, Layer-controlled band gap and anisotropic excitons in few-layer black phosphorus, Phys. Rev. B 89, 235319 (2014).

[14] V. Tran, R. Fei, and L. Yang, Quasiparticle energies, excitons, and optical spectra of few-layer black phosphorus, 2D Mater. 2, 044014 (2015).

[15] R. Schuster, J. Trinckauf, C. Habenicht, M. Knupfer, and B. Büchner, Anisotropic Particle-Hole Excitations in Black Phosphorus, Phys. Rev. Lett. 115, 026404 (2015).

[16] X. Wang, A. M. Jones, K. L. Seyler, V. Tran, Y. Jia, H. Zhao, H. Wang, L. Yang, X. Xu, and F. Xia, Highly anisotropic and robust excitons in monolayer black phosphorus, Nat. Nanotechnol. 10, 517 (2015).

[17] H. Yuan, X. Liu, F. Afshinmanesh, W. Li, G. Xu, J. Sun, B. Lian, A. G. Curto, G. Ye, Y. Hikita, Z. Shen, S. C. Zhang, X. Chen, M. Brongersma, H. Y. Hwang, and Y. Cui, Polarization-sensitive broadband photodetector using a black phosphorus vertical $p$ - $n$ junction, Nat. Nanotechnol. 10, 707 (2015).

[18] D. Y. Qiu, F. H. da Jornada, and S. G. Louie, Environmental screening effects in 2D materials: Renormalization of the bandgap, electronic structure, Nano Lett. 17, 4706 (2017).

[19] L. Li, J. Kim, C. Jin, G. J. Ye, D. Y. Qiu, F. H. da Jornada, Z. Shi, L. Chen, Z. Zhang, F. Yang, K. Watanabe, T. Taniguchi, W. Ren, S. G. Louie, X. H. Chen, Y. Zhang, and F. Wang, Direct observation of the layer-dependent electronic structure in phosphorene, Nat. Nanotechnol. 12, 21 (2017).

[20] C. S. Jung, F. Shojaei, K. Park, J. Y. Oh, H. S. Im, D. M. Jang, J. Park, and H. S. Kang, Red-to-ultraviolet emission tuning of two-dimensional gallium sulfide/selenide, ACS Nano 9, 9585 (2015).

[21] D. A. Bandurin, A. V. Tyurnina, G. L. Yu, A. Mishchenko, V. Zólyomi, S. V. Morozov, R. K. Kumar, R. V. Gorbachev, Z. R. Kudrynskyi, S. Pezzini, Z. D. Kovalyuk, U. Zeitler, K. S. Novoselov, A. Patanè, L. Eaves, I. V. Grigorieva, V. I. Fal'ko, A. K. Geim, and Y. Cao, High electron mobility, quantum Hall effect and anomalous optical response in atomically thin InSe, Nat. Nanotechnol. 12, 223 (2017).

[22] G. Shi and E. Kioupakis, Anisotropic spin transport and strong visible-light absorbance in few-layer $\mathrm{SnSe}$ and $\mathrm{GeSe}$, Nano Lett. 15, 6926 (2015).

[23] N. Sanders, D. Bayerl, G. Shi, K. A. Mengle, and E. Kioupakis, Electronic and optical properties of two-dimensional $\mathrm{GaN}$ from first-principles, Nano Lett. 17, 7345 (2017).

[24] AM 1.5 G solar spectrum from http://rredc.nrel.gov/solar/ spectra/am1.5/

[25] F. Bachhuber, J. von Appen, R. Dronskowski, P. Schmidt, T. Nilges, A. Pfitzner, and R. Weihrich, The extended stability range of phosphorus allotropes, Angew. Chem. Int. Ed. 53, 11629 (2014).

[26] J. C. Jamieson, Crystal structures adopted by black phosphorus at high pressures, Science 139, 1291 (1963).

[27] D. Scelta, A. Baldassarre, M. Serrano-Ruiz, K. Dziubek, A. B. Cairns, M. Peruzzini, R. Bini, and M. Ceppatelli, Interlayer bond formation in black phosphorus at high pressure, Angew. Chem. Int. Ed. 56, 14135 (2017).

[28] X. Li, J. Sun, P. Shahi, M. Gao, A. H. MacDonald, Y. Uwatoko, T. Xiang, J. B. Goodenough, J. Cheng, and J. Zhou, Pressureinduced phase transitions and superconductivity in a black phosphorus single crystal, Proc. Natl. Acad. Sci. USA 115, 9935 (2018).

[29] Z. Zhu and D. Tománek, Semiconducting Layered Blue Phosphorus: A Computational Study, Phys. Rev. Lett. 112, 176802 (2014).

[30] C. E. P. Villegas, A. S. Rodin, A. Carvalho, and A. R. Rocha, Two-dimensional exciton properties in monolayer semiconducting phosphorus allotropes, Phys. Chem. Chem. Phys. 18, 27829 (2016).

[31] J. L. Zhang, S. Zhao, C. Han, Z. Wang, S. Zhong, S. Sun, R. Guo, X. Zhou, C. D. Gu, K. D. Yuan, Z. Li, and W. Chen, Epitaxial growth of single layer blue phosphorus: A new phase of two-dimensional phosphorus, Nano Lett. 16, 4903 (2016).

[32] C. Gu, S. Zhao, J. L. Zhang, S. Sun, K. Yuan, Z. Hu, C. Han, Z. Ma, L. Wang, F. Huo, W. Huang, Z. Li, and W. Chen, Growth of quasi-free-standing single-layer blue phosphorus on tellurium monolayer functionalized Au(111), ACS Nano 11, 4943 (2017).

[33] J. P. Xu, J. Q. Zhang, H. Tian, H. Xu, W. Ho, and M. Xie, One-dimensional phosphorus chain and two-dimensional blue phosphorene grown on $\mathrm{Au}$ (111) by molecular-beam epitaxy, Phys. Rev. Mater. 1, 061002(R) (2017).

[34] E. Golias, M. Krivenkov, A. Varykhalov, J. Sánchez-Barriga, and $\mathrm{O}$. Rader, Band renormalization of blue phosphorus on $\mathrm{Au}$ (111), Nano Lett. 18, 6672 (2018).

[35] J. L. Zhang, S. Zhao, M. Telychko, S. Sun, X. Lian, J. Su, A. Tadich, D. Qi, J. Zhuang, Y. Zheng, Z. Ma, C. Gu, Z. Hu, Y. Du, J. Lu, Z. Li, and W. Chen, Reversible oxidation of blue phosphorus monolayer on $\mathrm{Au}(111)$, Nano Lett. 19, 5340 (2019).

[36] J. L. Zhang, S. Zhao, S. Sun, H. Ding, J. Hu, Y. Li, Q. Xu, X. Yu, M. Telychko, J. Su, C. Gu, Y. Zheng, X. Lian, Z. Ma, R. Guo, J. Lu, Z. Sun, J. Zhu, Z. Li, and W. Chen, Synthesis of monolayer blue phosphorus enabled by silicon intercalation, ACS Nano 14, 3687 (2020).

[37] W. Hittorf, Zur Kenntniss des Phosphors, Ann. Phys. Chem. 202, 193 (1865).

[38] H. Thurn and H. Krebs, Über struktur und eigenschaften der halbmetalle. XXII. Die kristallstruktur des hittorfschen phosphors, Acta Cryst. B 25, 125 (1969).

[39] M. Ruck, D. Hoppe, B. Wahl, P. Simon, Y. Wang, and G. Seifert, Fibrous red phosphorus, Angew. Chem. Int. Ed. 44, 7616 (2005).

[40] G. Schusteritsch, M. Uhrin, and C. J. Pickard, Single-layered Hittorf's phosphorus: A wide-bandgap high mobility 2D material, Nano Lett. 16, 2975 (2016).

[41] F. Xia, H. Wang, and Y. Jia, Rediscovering black phosphorus as an anisotropic layered material for optoelectronics and electronics, Nat. Commun. 5, 4458 (2014).

[42] L. Li, Y. Yu, G. J. Ye, Q. Ge, X. Ou, H. Wu, D. Feng, X. H. Chen, and Y. Zhang, Black phosphorus field-effect transistors, Nat. Nanotechnol. 9, 372 (2014).

[43] H. Liu, A. T. Neal, Z. Zhu, Z. Luo, X. Xu, D. Tománek, and P. D. Ye, Phosphorene: An unexplored 2D semiconductor with a high hole mobility, ACS Nano 8, 4033 (2014). 
[44] T. Cao, Z. Li, D. Y. Qiu, and S. G. Louie, Gate switchable transport and optical anisotropy in $90^{\circ}$ twisted bilayer black phosphorus, Nano Lett. 16, 5542 (2016).

[45] L. Zhang, H. Huang, B. Zhang, M. Gu, D. Zhao, X. Zhao, L. Li, J. Zhou, K. Wu, Y. Cheng, and J. Zhang, Structure and properties of violet phosphorus and its phosphorene exfoliation, Angew. Chem. Int. Ed. 59, 1074 (2020).

[46] L. V. Keldysh, Coulomb interaction in thin semiconductor and semimetal films, JETP Lett. 29, 658 (1979).

[47] P. Cudazzo, I. V. Tokatly, and A. Rubio, Dielectric screening in two-dimensional insulators: Implications for excitonic and impurity states in graphane, Phys. Rev. B 84, 085406 (2011).

[48] T. C. Berkelbach, M. S. Hybertsen, and D. R. Reichman, Theory of neutral and charged excitons in monolayer transition metal dichalcogenides, Phys. Rev. B 88, 045318 (2013).

[49] A. S. Rodin, A. Carvalho, and A. H. Castro Neto, Excitons in anisotropic two-dimensional semiconducting crystals, Phys. Rev. B 90, 075429 (2014).

[50] T. Olsen, S. Latini, F. Rasmussen, and K. S. Thygesen, Simple Screened Hydrogen Model of Excitons in Two-Dimensional Materials, Phys. Rev. Lett. 116, 056401 (2016).

[51] P. Giannozzi, S. Baroni, N. Bonini, M. Calandra, R. Car, C. Cavazzoni, D. Ceresoli, G. L. Chiarotti, M. Cococcioni, I. Dabo, A. D. Corso, S. de Gironcoli, S. Fabris, G. Fratesi, R. Gebauer, U. Gerstmann, C. Gougoussis, A. Kokalj, M. Lazzeri, L. Martin-Samos, N. Marzari, F. Mauri, R. Mazzarello, S. Paolini, A. Pasquarello, L. Paulatto, C. Sbraccia, S. Scandolo, G. Sclauzero, A. P. Seitsonen, A. Smogunov, P. Umari, and R. M. Wentzcovitch, QUANTUM ESPRESSO: A modular and open-source software project for quantum simulations of materials, J. Phys.: Condens. Matter 21, 395502 (2009).

[52] J. P. Perdew, K. Burke, and M. Ernzerhof, Generalized Gradient Approximation Made Simple, Phys. Rev. Lett. 77, 3865 (1996).

[53] M. S. Hybertsen and S. G. Louie, Electron correlation in semiconductors and insulators: Band gaps and quasiparticle energies, Phys. Rev. B 34, 5390 (1986).

[54] M. Rohlfing and S. G. Louie, Electron-hole excitations and optical spectra from first principles, Phys. Rev. B 62, 4927 (2000).

[55] J. Deslippe, G. Samzonidze, D. A. Strubbe, M. Jain, M. L. Cohen, and S. G. Louie, Berkeley GW: A massively parallel computer package for the calculation of the quasiparticle and optical properties of materials and nanostructures, Comput. Phys. Commun. 183, 1269 (2012).

[56] D. R. Hamann, Optimized norm-conserving Vanderbilt pseudopotentials, Phys. Rev. B 88, 085117 (2013).

[57] B. C. Shih, Y. Xue, P. Zhang, M. L. Cohen, and S. G. Louie, Quasiparticle Band Gap of ZnO: High Accuracy from the Conventional $G_{0} W_{0}$ Approach, Phys. Rev. Lett. 105, 146401 (2010).

[58] T. Y. Cai, S. C. Liu, S. Ju, C. Y. Liu, and G. Y. Guo, Multiferroic Double Perovskites $\mathrm{ScFe}_{1-x} \mathrm{Cr}_{x} \mathrm{O}_{3}(1 / 6 \leqslant x \leqslant 5 / 6)$ for Highly Efficient Photovoltaics and Spintronics, Phys. Rev. Appl. 8, 034034 (2017).

[59] J. Deslippe, M. Dipoppa, D. Prendergast, M. V. O. Moutinho, R. B. Capaz, and S. G. Louie, Electron-hole interaction in carbon nanotubes: Novel screening and exciton excitation spectra, Nano Lett. 9, 1330 (2009).
[60] T. Cao, M. Wu, and S. G. Louie, Unifying Optical Selection Rules for Excitons in Two Dimensions: Band Topology and Winding Numbers, Phys. Rev. Lett. 120, 087402 (2018).

[61] F. A. Ponce and D. P. Bour, Nitride-based semiconductors for blue and green light-emitting devices, Nature (London) 386, 351 (1997).

[62] C. D. Spataru, S. Ismail-Beigi, R. B. Capaz, and S. G. Louie, Theory and $A b$ Initio Calculation of Radiative Lifetime of Excitons in Semiconducting Carbon Nanotubes, Phys. Rev. Lett. 95, 247402 (2005).

[63] T. Langer, A. Chernikov, D. Kalincev, M. Gerhard, H. Bremers, U. Rossow, M. Koch, and A. Hangleiter, Room temperature excitonic recombination in GaInN/GaN quantum wells, Appl. Phys. Lett. 103, 202106 (2013).

[64] Y. Chen and S. Y. Quek, Tunable bright interlayer excitons in few-layer black phosphorus based van der Waals heterostructures, 2D Mater. 5, 045031 (2018).

[65] T. Korn, S. Heydrich, M. Hirmer, J. Schmutzler, and C. Schüller, Low-temperature photocarrier dynamics in monolayer $\mathrm{MoS}_{2}$, Appl. Phys. Lett. 99, 102109 (2011).

[66] D. Lagarde, L. Bouet, X. Marie, C. R. Zhu, B. L. Liu, T. Amand, P. H. Tan, and B. Urbaszek, Carrier and Polarization Dynamics in Monolayer $\mathrm{MoS}_{2}$, Phys. Rev. Lett. 112, 047401 (2014).

[67] H. Shi, R. Yan, S. Bertolazzi, J. Brivio, B. Gao, A. Kis, D. Jena, H. G. Xing, and L. Huang, Exciton dynamics in suspended monolayer and few-layer $\mathrm{MoS}_{2}$ 2D crystals, ACS Nano 7, 1072 (2013).

[68] C. Robert, D. Lagarde, F. Cadiz, G. Wang, B. Lassagne, T. Amand, A. Balocchi, P. Renucci, S. Tongay, B. Urbaszek, and $\mathrm{X}$. Marie, Exciton radiative lifetime in transition metal dichalcogenide monolayers, Phys. Rev. B 93, 205423 (2016).

[69] N. Kumar, Q. Cui, F. Ceballos, D. He, Y. Wang, and H. Zhao, Exciton-exciton annihilation in $\mathrm{MoSe}_{2}$ monolayers, Phys. Rev. B 89, 125427 (2014).

[70] G. Wang, L. Bouet, D. Lagarde, M. Vidal, A. Balocchi, T. Amand, X. Marie, and B. Urbaszek, Valley dynamics probed through charged and neutral exciton emission in monolayer $\mathrm{WSe}_{2}$, Phys. Rev. B 90, 075413 (2014).

[71] S. Mouri, Y. Miyauchi, M. Toh, W. Zhao, G. Eda, and K. Matsuda, Nonlinear photoluminescence in atomically thin layered $\mathrm{WSe}_{2}$ arising from diffusion-assisted exciton-exciton annihilation, Phys. Rev. B 90, 155449 (2014).

[72] C. G. Van de Walle and J. Neugebauer, First-Principles Calculations for Defects and Impurities: Applications to III-Nitrides, J. Appl. Phys. 95, 3851 (2004).

[73] S. B. Zhang and J. E. Northrup, Chemical Potential Dependence of Defect Formation Energies in GaAs: Application to Ga SelfDiffusion, Phys. Rev. Lett. 67, 2339 (1991).

[74] A. Förster, S. Gemming, G. Seifert, and D. Tománek, Chemical and electronic repair mechanism of defects in $\mathrm{MoS}_{2}$ monolayers, ACS Nano 11, 9989 (2017).

[75] D. Wang, D. Han, X. Li, N. Chen, D. West, V. Meunier, S. Zhang, and H. Sun, Charged defects in two-dimensional semiconductors of arbitrary thickness and geometry: Formulation and application to few-layer black phosphorus, Phys. Rev. B 96, 155424 (2017). 